\title{
Signifying the nonlocality of NOON states using Einstein-Podolsky-Rosen steering inequalities
}

\author{
R.Y. Teh, L. Rosales-Zárate, B. Opanchuk and M. D. Reid \\ Centre for Quantum and Optical Science, Swinburne University of Technology, Melbourne, Australia
}

\begin{abstract}
We construct Einstein-Podolsky-Rosen (EPR) steering signatures for the nonlocality of the entangled superposition state described by $\frac{1}{\sqrt{2}}\{|N\rangle|0\rangle+|0\rangle|N\rangle\}$, called the two-mode NOON state. The signatures are a violation of an EPR steering inequality based on an uncertainty relation. The violation confirms an EPR steering between the two modes and involves certification of an intermode correlation for number, as well as quadrature phase amplitude measurements. We also explain how the signatures certify an $N$ th order quantum coherence, so the system (for larger $N$ ) can be signified to be in a superposition of states distinct by a mesoscopic value of the two-mode quantum number difference. Finally, we examine the limitations imposed for lossy scenarios, discussing how experimental realisations may be possible for $N=2,3$.
\end{abstract}

\section{INTRODUCTION}

The generation and signification of a macroscopic quantum superposition state is an outstanding challenge. Schrodinger explained that according to quantum mechanics it is conceptually possible for a macroscopic system (like a cat) to become entangled with a microscopic one in such a way that a superposition of two macroscopically-distinct states is created [1 . Schrodinger pointed out the paradoxical nature of such a macroscopic system: A superposition of two macroscopically distinguishable states cannot be interpreted as being in one or the other of the states until measured. In realistic scenarios, couplings to external environments make the generation of macroscopic superposition states difficult but simpler mesoscopic realisations are feasible [2]4].

One of the most interesting realisations is the twomode NOON state [5-16]:

$$
\left|\psi_{\text {NOON }}\right\rangle=\frac{1}{\sqrt{2}}\left\{|N\rangle|0\rangle+e^{i \phi}|0\rangle|N\rangle\right\}
$$

Here, $N$ boson particles (or photons) are in a superposition of being either in the first mode (denoted $a$ ) or the second mode (denoted $b$ ). The modes may correspond to different spatial paths. Denoting the creation and destruction operators for the two modes by $\hat{a}, \hat{a}^{\dagger}$ and $\hat{b}$, $\hat{b}^{\dagger},|n\rangle|m\rangle$ is the eigenstate of numbers $\hat{n}_{a}=\hat{a}^{\dagger} \hat{a}$ and $\hat{n}_{b}=\hat{b}^{\dagger} \hat{b}$ with eigenvalues $n$ and $m$ respectively. Experiments have used spontaneous parametric down conversion to generate photonic NOON states for $N$ up to 5 [5, 8-13]. Recent experiments achieve Hong-Ou-Mandel interference with atoms (for $N=2$ ) [17] and proposals exist for Bose-Einstein condensates (BEC) [18]. NOON states are typically signified by way of interference fringes or fidelity [8, 13, 15].

As $N \rightarrow \infty$, the NOON state is a superposition of two states with macroscopically different values of quantum number $\hat{n}$ in each mode. While genuinely macroscopic systems (like a cat) would involve many degrees of freedoms (for example many modes) [19], the NOON state (similar to other single and two-mode states studied in the literature [3, 4]) nonetheless provides a simple model for the Schrodinger cat paradox, as $N \rightarrow \infty$. The NOON state superposition (1) can therefore elucidate aspects of the transition from microscopic to macroscopic. In order to quantify the transition, we refer to the state (1) as an " $N$-scopic superposition".

Our motivation is to investigate the nonlocality of the NOON state (or of an approximate NOON state that may be generated experimentally). While nonlocality between two microscopic systems (corresponding to $N=1$ ) has been experimentally certified using Bell inequalities [20], relatively little is known about nonlocality between more mesoscopic systems 21. In particular, it is an important goal to experimentally verify the nonlocality of an entangled state like that described by Schrodinger, where the system is in a superposition of two mesoscopically distinguishable states. In this paper, we derive a set of Einstein-Podolsky-Rosen (EPR) steering inequalities 22 24 based on the number-phase uncertainty relation

$$
\Delta \hat{n} \Delta \hat{P}^{N} \geq \frac{1}{2}\left|\left\langle\left[\hat{n}, \hat{P}^{N}\right]\right\rangle\right|
$$

where $\hat{n}$ is the mode number and $\hat{P}$ is the mode quadrature amplitude (defined below). We show how violation of these inequalities can be used to demonstrate the nonlocality of the NOON state for arbitrary $N$. By examining realistic scenarios for NOON states where losses are present, we suggest feasible tests for $N=2,3$.

The detection of an EPR steering nonlocality between two optical systems consisting of many photons has been experimentally verified [25], but this does not in itself imply the type of entangled state considered by Schrodinger: By contrast, we are able to show that the violation of the EPR steering inequalities as predicted for the NOON state certifies the $N$-scopic nature of the entanglement of (1), which involves a superposition of number states distinct by $N$ quanta.

EPR steering has been established as a distinct type of nonlocality, different to both Bell's nonlocality and entanglement [22, 23, 26]. "Steering" is the term used by Schrodinger [27] to describe the effect where an observer 
at one location can apparently change the quantum state at another - the effect Einstein called "spooky action-ata-distance" 28]. Some EPR steering and Bell inequalities have been derived for NOON states 29 31. For $N=1$ this led to the experimental verification of the Bell nonlocality of a single photon [31. More recently, steering inequalities for $N=1$ have been used to give conclusive proof of the "collapse of the wavefunction" [32, 33]. The EPR steering inequalities for larger $N$ may therefore open a way to investigate such effects for a mesoscopic superposition state.

Most steering and Bell inequalities derived to date use either number or quadrature phase amplitude measurements. The proposal of this paper combines number and quadrature phase amplitude measurements. This gives two advantages. First, the number measurements are useful in optimising violation of the inequalities for entangled two-mode systems over a range of field intensities where there is a perfect number correlation between the modes (as with NOON states). Second, the inequalities are based on variances and provide a simple method in nonideal scenarios with small losses to demonstrate that the nonlocality observed in the experiment is indeed due to a superposition of states distinct by $\sim N$ quanta.

Summary of paper: Our proposed EPR steering inequalities are derived in Sections II and III of this paper. For $N=2$ and $\phi \neq 0$ we show that a suitable signature for the steering nonlocality of a NOON state is the violation of the EPR steering inequality

$$
\Delta_{i n f} \hat{n}_{b} \Delta_{i n f}\left(\hat{P}_{b}^{2}\right) \geq\left|\left\langle\hat{C}_{b}\right\rangle\right|_{i n f} / 2
$$

where $\hat{C}_{b}=2 \hat{X}_{b, \pi / 4}-\hat{X}_{b}^{2}-\hat{P}_{b}^{2}$. Here we define the rotated quadrature phase amplitudes for mode $b$ as $\hat{X}_{b, \theta}=$ $\hat{X}_{b} \cos \theta+\hat{P}_{b} \sin \theta$ and $\hat{P}_{b, \theta}=-\hat{X}_{b} \sin \theta+\hat{P}_{b} \cos \theta$ where $\hat{X}_{b}=\hat{b}+\hat{b}^{\dagger}, \hat{P}_{b}=\left(\hat{b}-\hat{b}^{\dagger}\right) / i$. Also $\hat{X}_{a}=\hat{a}+\hat{a}^{\dagger}$, $\hat{P}_{a}=\left(\hat{a}-\hat{a}^{\dagger}\right) / i$. The $\Delta_{i n f} \hat{n}_{b}$ is the uncertainty in the prediction for $\hat{n}_{b}$ based on measurement of $\hat{n}_{a}$. Similarly $\Delta_{\text {inf }} \hat{P}_{b}^{2}$ is the uncertainty in $\hat{P}_{b}^{2}$ based on the measurement $\hat{X}_{a}$; and $\left|\left\langle\hat{C}_{b}\right\rangle\right|_{\text {inf }}$ is the magnitude of the mean value of $\hat{C}_{b}$ based on the measurement $\hat{X}_{a}$. An EPR steering inequality is obtained by replacing the quantities of an uncertainty relation (in this case (2)) with their predicted ("inferred") values [25, 34, 35]. In Section II, we summarise the Local Hidden State (LHS) Model developed by Wiseman, Jones and Doherty [22. Using the methods of Cavalcanti et al 23, we prove that (3) is a steering inequality the violation of which falsifies LHS models, so that steering of the mode $b$ (by measurements on the mode $a$ ) can be confirmed.

In Sections II and III, we provide similar inequalities for arbitrary $N$, including one for odd $N$ and $\phi=0$. Specifically, EPR steering of the mode $b$ is confirmed if

$$
E_{N}^{(p)}=\frac{\Delta_{i n f} \hat{n}_{b} \Delta_{i n f} \hat{P}_{b}^{N}}{\frac{1}{2}\left|\left\langle\left[\hat{n}_{b}, \hat{P^{N}}\right]\right\rangle\right|_{i n f}}<1
$$

or

$$
E_{N}^{(x)}=\frac{\Delta_{i n f} \hat{n}_{b} \Delta_{i n f} \hat{X}_{b}^{N}}{\frac{1}{2}\left|\left\langle\left[\hat{n}_{b}, \hat{X}_{b}^{N}\right]\right\rangle\right|_{i n f}}<1
$$

For the ideal NOON state, $\Delta_{i n f} \hat{n}_{b}=0$ and the usefulness of the inequality depends on whether the denominator is nonzero. We show that the first criterion is useful provided $\cos \phi \neq 0$ for $N$ odd or $\sin \phi \neq 0$ for $N$ even, and the second criterion is useful for all $N$ provided $\sin \phi \neq 0$. We explain in Section VII for $N$ up to 3 how the denominator of the inequality can be measured via homodyne detection. For $N=1$ the inequality is becomes straightforwardly

$$
\Delta_{i n f} \hat{n}_{b} \Delta_{i n f} \hat{P}_{b}<\left|\left\langle\hat{X}_{b}\right\rangle\right|_{i n f} / 2
$$

The cases of $N=1$ and $N=2$ are analysed in detail in Sections IV and V. The explanation of how the steering inequalities signify an $N$-scopic superposition state is given in Section VIII.

The inequalities (35) involve measurement of number $\left(\hat{n}_{a}, \hat{n}_{b}\right)$ and hence have the drawback of low detection efficiencies (in the photonic case). In the first instance, we propose that the correlation be established by postselection of the events where a total of $N$ quanta (photons) are detected at the sites of both modes. A second problem is distinguishing between the detection of two and one photons at a given site. Here, beam splitters or photon number-resolving detectors could be used [10, 13] in conjunction with postselection over events where a total $N$ photons is counted. The measurement of observables $\hat{X}^{N}, \hat{P}^{N}$ is achieved via optical homodyne techniques that are highly efficient. Nonetheless, we explain in Sections VI, VII and IX that losses have a significant effect (measurement efficiencies of $\eta>0.94$ are required for $N=3$ ) and that care needs to be taken to avoid possible loopholes created by asymmetrical losses for the number and quadrature measurements.

\section{EPR STEERING INEQUALITIES BASED ON UNCERTAINTY RELATIONS}

In this Section we give the formal derivation of the EPR steering criteria summarised in the Introduction I. We show in Section III how we can use the inequalities to detect EPR steering for a NOON state.

\section{A. EPR steering inequalities}

EPR steering is verified as a failure of Local Hidden State models (LHS). The LHS model was pioneered in the papers by Wiseman, Jones and Doherty [22] and is based on the Local Hidden Variable models considered by Bell [36]. We define two subsystems $A$ and $B$ and consider space-like separated measurements on each of them. 
The measurements are described quantum mechanically by observables $\hat{X}_{A}(\theta)$ and $\hat{X}_{B}(\phi)$ (respectively) and the outcomes are given by the numbers $X_{A}(\theta)$ and $X_{B}(\phi)$ (written without the "hats"). Here $\theta$ and $\phi$ denote the measurement choice at the locations $A$ and $B$. To prove Bell's nonlocality, one falsifies a description of the statistics based on a Local Hidden Variable model, where the averages are given as

$$
\left\langle X_{B}(\phi) X_{A}(\theta)\right\rangle=\int_{\lambda} d \lambda P(\lambda)\left\langle X_{B}(\phi)\right\rangle_{\lambda}\left\langle X_{A}(\theta)\right\rangle_{\lambda}
$$

Here $\int_{\lambda} P(\lambda) d \lambda=1$ so that the $P(\lambda)$ is a probability density (or probability if the integral is replaced by a discrete summation, as explained in Bell's papers [36]). The $\lambda$ denotes a set of variables $\{\lambda\}$ that take the role of the hidden variables as postulated in Bell's model. The $\left\langle X_{A}\right\rangle_{\lambda}$ denotes the average of the results $X_{A}$ for the system in the particular hidden variable state denoted by $\lambda$; and similarly for $\left\langle X_{B}\right\rangle_{\lambda}$. The $P(\lambda)$ is independent of the $\theta$ and $\phi$. The factorisation that occurs for the moments in the integrand is due to the assumption of "locality" 36 .

To prove EPR steering of subsystem $B$, we need to falsify a description of the statistics based on a Local Hidden State (LHS) model where the averages are given as 22, 23.

$$
\left\langle X_{B}(\phi) X_{A}(\theta)\right\rangle=\int_{\lambda} d \lambda P(\lambda)\left\langle X_{B}(\phi)\right\rangle_{\lambda, \rho}\left\langle X_{A}(\theta)\right\rangle_{\lambda}
$$

Here an extra condition is placed on the average $\left\langle X_{B}\right\rangle_{\lambda}$. The $\rho$ subscript denotes that the average is to be consistent with that of a quantum density operator $\rho_{\lambda}^{B}$. This is the case for all choices $\phi$ of measurement at $B$. For example, if $X_{B}(\theta)=X_{B}$ and $X_{B}(\pi / 2)=P_{B}$ then the statistics for the LHS model must be consistent with a local uncertainty principle namely $\left\langle\left(X_{B}-\left\langle X_{B}\right\rangle\right)^{2}\right\rangle_{\lambda}\left\langle\left(P_{B}-\right.\right.$ $\left.\left.\left\langle P_{B}\right\rangle\right)^{2}\right\rangle_{\lambda} \geq 1$. The $\rho_{\lambda}^{B}$ is an example of a Local Quantum State (for site $B$ ). No such constraint is made for the moments $\left\langle X_{A}(\theta)\right\rangle_{\lambda}$, written without the subscript.

In this paper we consider three quantum observables defined through the uncertainty relation:

$$
\Delta \hat{\sigma}_{B}^{X} \Delta \hat{\sigma}_{B}^{Y} \geq\left|\left\langle\hat{\sigma}_{B}^{Z}\right\rangle\right| / 2
$$

Following the approach given in Refs. [25, 34] used to derive a criterion for the EPR paradox [26] and also for EPR steering [22, 25], we consider the average conditional uncertainty $\Delta_{i n f} \sigma_{B}^{X}$ defined by

$$
\left(\Delta_{i n f} \hat{\sigma}_{B}^{X}\right)^{2}=\sum_{x_{j}^{A}} P\left(x_{j}^{A}\right)\left(\Delta\left(\sigma_{B}^{X} \mid x_{j}^{A}\right)\right)^{2}
$$

Here, we denote the possible results of a measurement $\hat{X}_{A}$ at $A$ by $\left\{x_{j}^{A}\right\} . P\left(x_{j}^{A}\right)$ is the probability for obtaining the result $x_{j}^{A}$. The uncertainty 10 is a measure of the (average) uncertainty in the inferred value (which we take to be the mean of the conditional distribution $\left.P\left(\sigma_{B}^{X} \mid x_{j}^{A}\right)\right)$ for a measurement $\hat{\sigma}_{B}^{X}$ at $B$ given a measurement $\hat{X}_{A}$ at $A$. Specifically, $\left(\Delta\left(\sigma_{B}^{X} \mid x_{j}^{A}\right)\right)^{2}$ is the variance of the conditional distribution $P\left(\sigma_{B}^{X} \mid x_{j}^{A}\right)$. We define similarly

$$
\left(\Delta_{i n f} \hat{\sigma}_{B}^{Y}\right)^{2}=\sum_{y_{j}^{A}} P\left(y_{j}^{A}\right)\left(\Delta\left(\sigma_{B}^{X} \mid y_{j}^{A}\right)\right)^{2}
$$

noting that the $\left\{y_{j}\right\}$ is the set of results for a measurement $\hat{Y}_{A}$ made at $A$ to infer the value of the measurement of $\hat{\sigma}_{B}^{Y}$ at $B$. Further, we define an (average) inferred value for the modulus of the mean of measurement of $\hat{\sigma}_{B}^{Z}$ given a measurement $\hat{Z}_{A}$ at $A$ as

$$
\left|\left\langle\hat{\sigma}_{B}^{Z}\right\rangle\right|_{i n f}=\sum_{z_{j}^{A}} P\left(z_{j}^{A}\right)\left|\left\langle\sigma_{B}^{Z}\right\rangle_{z_{j}^{A}}\right|
$$

Here $\left\langle\sigma_{B}^{Z}\right\rangle_{z_{j}^{A}}$ is the mean of the conditional distribution $P\left(\sigma_{B}^{Z} \mid z_{j}^{A}\right)$ and the $\left\{z_{j}\right\}$ is the set of values for a measurement $\hat{Z}_{A}$ at $A$, that we use to infer outcomes for $\hat{\sigma}_{B}^{Z}$. Using these definitions, we can prove the following result 35 .

Result (1): - The EPR steering inequality

The LHS model (8) implies the inequality

$$
\left(\Delta_{i n f} \hat{\sigma}_{B}^{X}\right)\left(\Delta_{i n f} \hat{\sigma}_{B}^{Y}\right) \geq\left|\left\langle\hat{\sigma}_{B}^{Z}\right\rangle\right|_{i n f} / 2
$$

Hence, violation of this inequality (called an EPR steering inequality) implies failure of the LHS model (Eq. (8), and therefore steering of system $B$ by (measurements at $A$ ). The proof is given in the Appendix A.

\section{STEERING INEQUALITIES FOR THE NOON STATE}

To arrive at a steering signature for a NOON state, we consider the three observables for each mode: number $\hat{n}$, and the two quadrature phase amplitudes $\hat{X}$ and $\hat{P}$. Specifically: $\hat{n}_{a}=\hat{a}^{\dagger} \hat{a}, \hat{X}_{a}=\hat{a}+\hat{a}^{\dagger}$ and $\hat{P}_{a}=(\hat{a}-$ $\left.\hat{a}^{\dagger}\right) / i$, and $\hat{n}_{b}=\hat{b}^{\dagger} \hat{b}, \hat{X}_{b}=\hat{b}+\hat{b}^{\dagger}$ and $\hat{P}_{b}=\left(\hat{b}-\hat{b}^{\dagger}\right) / i$. Where the notation is clear, we omit the "hat" for these operators. Using the Result (1) given by Eq. (13), we can write down EPR steering criteria associated with the three observables: We certify EPR steering (of $B$ by $A$ ) if either one of the following hold:

$$
\Delta_{i n f} n_{b} \Delta_{i n f}\left(P_{b}^{N}\right)<\left|\left\langle\left[n_{b}, P_{b}^{N}\right]\right\rangle\right|_{i n f} / 2
$$

and

$$
\Delta_{i n f} n_{b} \Delta_{i n f}\left(X_{b}^{N}\right)<\left|\left\langle\left[n_{b}, X_{b}^{N}\right]\right\rangle\right|_{i n f} / 2
$$

Here, $\Delta_{i n f} n_{b}$ refers to the average uncertainty of the result for $n_{b}$ given a measurement $\hat{O}_{n}$ at $A$, as defined by 
10. Similarly, $\Delta_{i n f} P_{b}^{N}$ refers to the average uncertainty of the result for $P_{b}^{N}$ given a measurement $\hat{O}_{p}$ at $A$. The $\Delta_{\text {inf }} X_{b}^{N}$ refers to the average uncertainty of the result for $X_{b}^{N}$ given a measurement $\hat{O}_{x}$ at $A$. The $|\langle\hat{C}\rangle|_{\text {inf }}$ where $\hat{C}=\left[n_{b}, P_{b}^{N}\right]$ (or $\left[n_{b}, X_{b}^{N}\right]$ ) is defined similarly, by (12), as the average value of the modulus of the expectation value of $\hat{C}$ conditioned on a measurement $\hat{O}_{c}$ at $A$. The steering inequalities of this paper take $\hat{O}_{n}=\hat{n}_{a}$, $\hat{O}_{p}=\hat{X}_{a}, \hat{O}_{x}=\hat{X}_{a}$ and $\hat{O}_{c}=\hat{X}_{a}$. The motivation for this choice is explained in Section IV.

To evaluate the right side of the inequalities 14 15, we determine the commutation relations: $[n, X]=-i P$ and $[n, P]=i X$. By ordering the $P$ 's to be always on the left of the $X$ 's and since $[X, P]=2 i$, we arrive at the commutation relation $\left[X, P^{k}\right]=2 i k P^{k-1}$. It can be shown that $\left[n, P^{N}\right]=i N\left\{P^{N-2}[P X+(N-1) i]\right\}$ and $\left[n, X^{N}\right]=-i N\left\{X^{N-2}[X P-(N-1) i]\right\}$. We use this result to further evaluate the right side of the steering inequalities. Most generally, the right side of the steering inequality (14) can be written

$$
\left|\left\langle\left[n_{b}, P_{b}^{N}\right]\right\rangle\right|_{i n f}=N\left|\left\langle P_{b}^{N-1} X_{b}+i(N-1) P_{b}^{N-2}\right\rangle\right|_{\text {inf }}
$$

so that the procedure is to measure the modulus of the expectation value of the measurement $\hat{C}=P_{b}^{N-1} X_{b}+$ $i(N-1) P_{b}^{N-2}$ made on mode $b$, given a specific result for a measurement $\hat{O}_{c}$ is made on mode $a$, and then take the weighted average as defined by $(12)$. We discuss methods for measuring $P_{b}^{N-1} X_{b}$ where $N=1,2,3$ in Section VII below.

To investigate whether the steering inequalities will be useful for the NOON states (1) with phase $\phi$, we evaluate the prediction for the right side of the steering inequality 14 in the general NOON case. We will take $\hat{O}_{c}$ to be the measurement $X_{a}$ and denote the result of that measurement by $x$. We find

$$
\begin{aligned}
\left|\left\langle\left[n_{b}, P_{b}^{N}\right]\right\rangle\right|_{\text {inf }}= & N \mid\left\langle\left. P_{b}^{N-2}\left(P_{b} X_{b}+(N-1) i\right\rangle\right|_{\text {inf }}\right. \\
= & N \mid\left(\left\langle b^{N}\right\rangle+\left.(-1)^{N+1}\left\langle b^{\dagger N}\right\rangle\right|_{\text {inf }}\right. \\
= & N \sqrt{N !}\left[\frac{\left[e^{i \phi}+(-1)^{N+1} e^{-i \phi}\right]}{2} \mid\right. \\
& \times \int_{-\infty}^{\infty}|\langle x \mid 0\rangle\langle x \mid N\rangle| d x
\end{aligned}
$$

where $|x\rangle$ are the eigenstates of $X$. The cases $N=1$ and $N=2$ are presented in the Sections IV and V below. We find similarly

$$
\begin{aligned}
\left|\left\langle\left[n_{b}, X_{b}^{N}\right]\right\rangle\right|_{\text {inf }} & =N\left|\left\langle X_{b}^{N-2}\left[X_{b} P_{b}-(N-1) i\right]\right\rangle\right|_{\text {inf }} \\
& =N\left|-\left\langle b^{N}\right\rangle+\left\langle\left(b^{\dagger}\right)^{N}\right\rangle\right|_{\text {inf }} \\
& =N \sqrt{N !}|\sin \phi| \int_{-\infty}^{\infty}|\langle x \mid 0\rangle\langle x \mid N\rangle| d x
\end{aligned}
$$

Now we determine when each of the steering criteria (14) and (15) will be useful. For the NOON state, the mode numbers are always correlated, and we observe that $\Delta_{i n f} n_{b}=0$. Hence either of the steering criteria (14) and 15. will be effective to detect steering in NOON states, provided that the right side of the inequality is not zero, and provided the variances $\Delta_{i n f}\left(X_{b}^{N}\right), \Delta_{i n f}\left(P_{b}^{N}\right)$ are finite. Since the integral $\int_{-\infty}^{\infty}|\langle x \mid 0\rangle\langle x \mid N\rangle| d x$ is nonzero for the NOON state, we see from the expressions (17) and (18) that the condition for the right side of the inequalities (14) and 15) to be nonzero is: for $N$ odd, $\cos \phi \neq 0$ and $\sin \phi \neq 0$ respectively; for $N$ even, $\sin \phi \neq 0$ in both cases.

To summarise, we rewrite the EPR steering criteria (14) and (15) as

$$
E_{N}^{(p)}=\frac{\Delta_{i n f} n_{b} \Delta_{i n f} P_{b}^{N}}{\frac{1}{2}\left|\left\langle\left[n_{b}, P_{b}^{N}\right]\right\rangle\right|_{i n f}}<1
$$

and

$$
E_{N}^{(x)}=\frac{\Delta_{i n f} n_{b} \Delta_{i n f} X_{b}^{N}}{\frac{1}{2}\left|\left\langle\left[n_{b}, X_{b}^{N}\right]\right\rangle\right|_{i n f}}<1
$$

Steering is obtained if $E_{N}^{(x / p)}<1$. Either criterion is sufficient to certify an EPR paradox, or EPR steering. For the NOON state $\left|\psi_{\text {NOON }}\right\rangle=\frac{1}{\sqrt{2}}\left\{|N\rangle|0\rangle+e^{i \phi}|0\rangle|N\rangle\right\}$ the first criterion is useful provided $\cos \phi \neq 0$ for $N$ odd or $\sin \phi \neq 0$ for $N$ even, and the second criterion is useful for all $N$ provided $\sin \phi \neq 0$. We comment that the right side of the steering inequalities (14) and $(15)$ needs to be measured in the experiment. We examine how this can be done below in Section VII, finding that cases of low $N$ are much more accessible to experiment. We also point out that except where $N=1$ or 2 , the equivalence of the first two lines in equations $(17)$ and $(18)$ holds only for the expectation values as calculated for the ideal NOON state (1).

In Section VI, we will evaluate predictions for non-ideal case where loss is present. To complete the prediction for the steering inequalities with loss present, we also need to calculate $\Delta_{i n f}\left(P^{N}\right), \Delta_{i n f}\left(X^{N}\right)$. In this paper, we use $\hat{O}_{x}=\hat{O}_{p}=\hat{X}_{a}$ as the measurement on mode $a$. As above, we take $x$ to be the result of the measurement $X_{a}$. 




Figure 1: Predictions for EPR steering of the NOON states. EPR steering is observed when $E_{N}^{(p)}<1$. It is assumed that the two-mode NOON state is created and that each mode is then (independently) subjected to losses. Loss at each mode is modeled by a beam splitter coupling as described in Sec. VI. Here the beam splitter transmission efficiencies are $\eta=\eta_{a}=\eta_{b}$. We select the NOON state (1) with $\phi=0$ for $N$ odd and $\phi=\pi / 2$ for $N$ even.

We evaluate

$$
\begin{aligned}
\Delta_{\text {inf }}^{2}\left(P_{b}^{N}\right) & =\int_{-\infty}^{\infty} P(x)\left\{\Delta\left(P_{b}^{N} \mid x\right)\right\}^{2} d x \\
& =\int_{-\infty}^{\infty} P(x)\left[\left\langle P_{b}^{2 N}\right\rangle_{x}-\left\langle P_{b}^{N}\right\rangle_{x}^{2}\right] d x
\end{aligned}
$$

where $\langle\ldots\rangle_{x}$ denotes the expectation value conditioned on the result $x$, as defined for $(12)$. The $\left\langle P_{b}^{2 N}\right\rangle_{x}$ and $\left\langle P_{b}^{N}\right\rangle_{x}$ can be expressed in terms of the momentum representation functions $\langle p \mid N\rangle$ as shown in Appendix B Similarly

$$
\begin{aligned}
\Delta_{i n f}^{2}\left(X_{b}^{N}\right) & =\int_{-\infty}^{\infty} P(x)\left\{\Delta\left(X_{b}^{N} \mid x\right)\right\}^{2} d x \\
& =\int_{-\infty}^{\infty} P(x)\left[\left\langle X_{b}^{2 N}\right\rangle_{x}-\left\langle X_{b}^{N}\right\rangle_{x}^{2}\right] d x
\end{aligned}
$$

The $\left\langle X_{b}^{N}\right\rangle_{x}$ and $\left\langle X_{b}^{2 N}\right\rangle_{x}$ can be solved in terms of the harmonic oscillator wavefunctions $\langle x \mid N\rangle(27)$ as shown in Appendix $\mathrm{B}$ and explained for $N=1,2$ below. We have introduced the shorthand notation $\Delta^{2} x \equiv(\Delta x)^{2}$ to avoid overuse of brackets. We have solved for the effect of loss on the NOON states using the methods outlined in Section VI and the results for the steering inequalities are plotted in Figure 1.

\section{SPECIAL CASE OF $N=1$}

Steering for the case of $N=1$ has been proposed by Jones and Wiseman [32] and experimentally achieved by Fuwa et al [33. The inequalities used in those papers verified steering in the high efficiency limit based on homodyne detection, thus giving a firm experimental proof of the nonlocality of the NOON $(N=1)$ state. Here, we outline the application of the steering inequalities (14) and (15) for this case.

For $N=1$, the relevant Heisenberg uncertainty relations are $\Delta n \Delta P \geq|\langle X\rangle| / 2$ and $\Delta n \Delta X \geq|\langle P\rangle| / 2$. We see from (13) that a criterion sufficient to certify EPR steering of mode $b$ by measurements on mode $a$ is

$$
\Delta_{i n f} n_{b} \Delta_{i n f} P_{b}<\left|\left\langle X_{b}\right\rangle\right|_{i n f} / 2
$$

The inequality $\Delta_{i n f} n_{b} \Delta_{i n f} X_{b}<\left|\left\langle P_{b}\right\rangle\right|_{\text {inf }} / 2$ is also a steering criterion. Note we can also define the corresponding criteria for steering of the $a$ mode by interchanging the $a$ and $b$ indices. The quantities have been defined above in Section II and III.

The choice of measurements $\hat{O}_{n}, \hat{O}_{c}, \hat{O}_{p}, \hat{O}_{x}=\hat{X}_{a}$ to be made on the mode $a$ (as defined for equations (14) and (15)) is generally so as to optimise the criterion for a given state, but is otherwise not explicitly specified in the criterion. Here, the choice of $\hat{O}_{n}=\hat{n}_{a}$ is crucial because it takes advantage of the correlation of number between the two modes of the NOON state, to allow precisely that $\Delta_{i n f} n_{b}=0$. The criterion (23) is then predicted to be satisfied for any finite $\Delta_{i n f} P_{b}$, provided $\left|\left\langle X_{b}\right\rangle\right|_{\text {inf }} \neq 0$. For the choice of $\hat{O}_{c}, \hat{O}_{p}$, we focus on quadrature phase amplitude measurements because they are readily measurable experimentally. For $\Delta_{i n f} P_{b}$, we select $\hat{O}_{p}=\hat{X}_{a}$, but we note in the Appendices (BD) that the result is not particularly sensitive to this choice. On the other hand, without a suitable measurement on mode $a,\left|\left\langle X_{b}\right\rangle\right|_{\text {inf }}$ will vanish. We find below that the measurement $\hat{X}_{a}$ on $a$ does not completely collapse the state $b$, and the resulting superposition predicts a nonzero result for $\left|\left\langle X_{b}\right\rangle\right|_{\text {inf }}$. With this motivation, we take $\hat{O}_{n}=\hat{n}_{a}, \hat{O}_{p}=\hat{X}_{a}, \hat{O}_{x}=\hat{X}_{a}$ and $\hat{O}_{c}=\hat{X}_{a}$.

We examine the NOON state $|\psi\rangle=\frac{1}{\sqrt{2}}\{|N\rangle|0\rangle+$ $|0\rangle|N\rangle\} \quad(\phi=0)$ and restrict therefore to the steering criterion 23. The measurement of $n_{a}$ will enable a perfectly accurate prediction for the number $n_{b}$, so that $\Delta_{i n f} n=0$. Taking $\hat{O}_{c}=X_{a}$ we evaluate the mean of $X_{b}$ (or $P_{b}$ ) at $b$, given a result $x$ for measurement of $X_{a}$ at $A$. This enables us to evaluate $\left|\left\langle X_{b}\right\rangle\right|_{\text {inf }}$ and $\left|\left\langle P_{b}\right\rangle\right|_{\text {inf }}$ for a valid steering criterion. If we measure $X_{a}$ with result $x$, the normalised reduced wave function is (we denote the eigenstate of $X$ for mode $a$ by $|x\rangle)$

$$
|\psi\rangle_{x}=\frac{\langle x \mid N\rangle|0\rangle+\langle x \mid 0\rangle|N\rangle}{\sqrt{|\langle x \mid N\rangle|^{2}+|\langle x \mid 0\rangle|^{2}}}
$$


Thus we write the reduced density operator as

$$
\begin{aligned}
\rho_{\text {red, } x}= & \frac{1}{2 P(x)}\left\{|\langle x \mid N\rangle|^{2}|0\rangle\left\langle\left. 0|+|\langle x \mid 0\rangle\right|^{2} \mid N\right\rangle\langle N|\right. \\
& +\langle 0 \mid x\rangle\langle x \mid N\rangle|0\rangle\langle N|+\langle N \mid x\rangle\langle x \mid 0\rangle| N\rangle\langle 0|\}
\end{aligned}
$$

where the probability distribution for obtaining a result $x$ for $X_{a}$ is

$$
P(x)=\frac{1}{2}\left\{|\langle x \mid 0\rangle|^{2}+|\langle x \mid N\rangle|^{2}\right\}
$$

Here $\langle x \mid n\rangle$ are the standard oscillator wave functions

$$
\langle x \mid n\rangle=\left(\sqrt{\pi} 2^{n} n !\right)^{-\frac{1}{2}} \frac{2^{\frac{1}{4}}}{\sqrt{c}} e^{-\frac{x^{2}}{c^{2}}} H_{n}\left(\frac{\sqrt{2}}{c} x\right)
$$

involving Hermite polynomials $H_{n}$ and derived using that $\hat{x}=\frac{c}{2}\left(\hat{a}+\hat{a}^{\dagger}\right), \hat{p}=\frac{c}{2 i}\left(\hat{a}-\hat{a}^{\dagger}\right)$. In this paper we have taken $c=2$. Now we see that the mean for $X_{b}$ given the result $x$ for $X_{a}$ is

$$
\begin{aligned}
\left\langle X_{b}\right\rangle_{x}= & \operatorname{Tr}\left(\rho_{\text {red, }, x} X_{b}\right) \\
= & \frac{1}{2 P(x)}\left\{\langle 0 \mid x\rangle\langle x \mid N\rangle\left\langle N\left|X_{b}\right| 0\right\rangle\right. \\
& \left.+\langle N \mid x\rangle\langle x \mid 0\rangle\left\langle 0\left|X_{b}\right| N\right\rangle\right\}
\end{aligned}
$$

and similarly

$$
\begin{aligned}
\left\langle P_{b}\right\rangle_{x}= & \operatorname{Tr}\left(\rho_{\text {red,x }} P_{b}\right) \\
= & \frac{1}{2 P(x)}\left\{\langle 0 \mid x\rangle\langle x \mid N\rangle\left\langle N\left|P_{b}\right| 0\right\rangle \mid\right. \\
& \left.+\langle N \mid x\rangle\langle x \mid 0\rangle\left\langle 0\left|P_{b}\right| N\right\rangle\right\}
\end{aligned}
$$

In fact the mean $\left\langle X_{b}\right\rangle_{x}$ will be nonzero only for $N=$ 1 , in which case the steering criterion (23) is satisfied because $\Delta_{i n f} n_{b}=0$ (and $\Delta_{i n f} P_{b} \neq \infty$ ). Hence, the inequality (23) is a suitable steering criterion for $N=1$. Specifically, following the definition (12), we evaluate

$$
\left|\left\langle X_{b}\right\rangle\right|_{\text {inf }}=\int_{-\infty}^{\infty} P(x)\left|\left\langle X_{b}\right\rangle_{x}\right| d x=\sqrt{\frac{2}{\pi}}
$$

where $\left\langle X_{b}\right\rangle_{x}$ is the conditional quantity between two modes, as defined in (12). To complete the prediction for the steering inequality, we calculate a suitable value for $\Delta_{i n f} P_{b}$ by selecting the measurement at $A$ to be $X_{a}$. We denote the result of that measurement by $x$. Then the reduced density operator is $\rho_{\text {red,x }}$ as above, which for $N=1$ gives

$$
\left(\Delta\left(P_{b} \mid x\right)\right)^{2}=\frac{1}{2 P(x)}\left\{|\langle x \mid 1\rangle|^{2}+3|\langle x \mid 0\rangle|^{2}\right\}
$$

and thus

$$
\begin{aligned}
\Delta_{i n f}^{2} P_{b} & =\int_{-\infty}^{\infty} P(x)\left\{\Delta\left(P_{b} \mid x\right)\right\}^{2} d x \\
& =\frac{1}{2} \int_{-\infty}^{\infty}\left\{|\langle x \mid 1\rangle|^{2}+3|\langle x \mid 0\rangle|^{2}\right\}=2
\end{aligned}
$$

where $\Delta^{2} x \equiv(\Delta x)^{2}$. We obtain an EPR steering when $E_{1}^{(p)} \equiv \frac{\Delta_{i n f} n_{b} \Delta_{i n f} P_{b}}{\left|\left\langle X_{b}\right\rangle\right|_{i n f} / 2}<1$. For the ideal NOON state with no losses, $E_{1}^{(p)}=0$ and the steering is always detectable via this criterion. The situation with loss is studied in Section VI and presented in Figure 1. Efficiencies $\eta>$ 0.92 are required to detect the steering.

\section{SPECIAL CASE OF $N=2$}

We now examine the details for the NOON state with $N=2$ which represents an important case potentially accessible to experiment, in view of recent advances 13 , 17, 33. Firstly, $\left[n, X^{2}\right]=-i(X P+P X)=-2 i X P-2=$ $2\left(a^{\dagger 2}-a^{2}\right)$. Similarly, $\left[n, P^{2}\right]=i(X P+P X)=-2\left(a^{\dagger 2}-\right.$ $\left.a^{2}\right)$. The steering criteria are

$$
\Delta_{i n f} n \Delta_{i n f}\left(X^{2}\right)<\left|\left\langle a^{\dagger 2}-a^{2}\right\rangle\right|_{i n f}
$$

and

$$
\Delta_{i n f} n \Delta_{i n f}\left(P^{2}\right)<\left|\left\langle a^{\dagger 2}-a^{2}\right\rangle\right|_{i n f}
$$

For the NOON state (1) with $N=2$ we obtain

$$
\begin{aligned}
\left|\left\langle a^{\dagger 2}-a^{2}\right\rangle\right|_{\text {inf }} & =\int P(x)\left|\left\langle\hat{a}^{2} \mid x\right\rangle-\left\langle\left(\hat{a}^{\dagger}\right)^{2} \mid x\right\rangle\right| d x \\
& =\sqrt{2}|\sin \phi| \int|\langle x \mid 0\rangle\langle x \mid 2\rangle| d x \\
& =2 \sqrt{\frac{2}{e \pi}}|\sin \phi|=0.968|\sin \phi|
\end{aligned}
$$

Both the steering criteria (33) and (34) become useful for the NOON state with $\phi=\pi / 2$. We show in Appendix $\mathrm{B}$ by integration of the Hermite polynomials that $\Delta_{i n f}\left(X^{2}\right)=3.18$ and $\Delta_{i n f}\left(P^{2}\right)=3.18$. For the ideal case with no detection loss, $\Delta_{\text {inf }} n=0$ and the steering for the NOON state with $N=2$ is detectable using either criterion. Comparing with the results for $N=1$, we see that the prediction for the ratio of the right- to left-sides of the steering inequalities decreases for $N=2$. We expect the criteria will be more difficult to satisfy at higher $N$ in non-ideal cases. Details of the calculations for arbitrary $N$ are given in Appendices B and C. The effect of the losses is studied below in Section VI and the results are shown in Figure 1. 


\section{INCLUDING LOSSES}

Signatures of the NOON state superposition are known to be fragile to losses. We examine the effect of loss on the signatures proposed here, by using a simple model for loss. We couple each mode $a$ and $b$ to second independent fields taken as single modes and initially in independent vacuum states, following the beam splitter model introduced for the study of the decoherence of a macroscopic superposition state by Yurke and Stoler [3]. We thus evaluate the moments of detected fields with boson operators $a_{\text {det }}, b_{\text {det }}$ given by

$$
\begin{aligned}
& a_{\text {det }}=\sqrt{\eta_{a}} a+\sqrt{1-\eta_{a}} a_{v} \\
& b_{\text {det }}=\sqrt{\eta_{b}} b+\sqrt{1-\eta_{b}} b_{v}
\end{aligned}
$$

Here the $a_{v}$ and $b_{v}$ are destruction operators for independent external vacuum modes that couple to the modes of the NOON state. These external modes model the presence of an external environment into which quanta can be lost from the $a$ and $b$ modes. The amount of coupling for each mode is determined by the efficiency factors $\eta_{a}$ and $\eta_{b}$ respectively. The $\eta_{A / B}=1$ indicates zero loss; low $\eta_{A / B}$ indicates high loss. The model is effective for optical NOON states where thermal noise can be neglected. The full calculation is explained in Appendix D. We find for $N=1$ and $\phi=0$

$$
\begin{aligned}
E_{1}^{(p)} & \equiv \frac{\Delta_{i n f} n \Delta_{i n f} P}{|\langle X\rangle|_{i n f} / 2} \\
& =2\left[\frac{\eta_{b}\left(\eta_{a}+\eta_{b}-2\right)}{2\left(\eta_{a}-2\right)}\left(1+\eta_{b}\right)\right]^{\frac{1}{2}} /\left[\sqrt{\frac{2}{\pi}} \sqrt{\eta_{a} \eta_{b}}\right]
\end{aligned}
$$

The expressions for higher $N$ are more complex but are explained in Appendix D and evaluated numerically. Figure 1 shows $E_{N}^{(p)}$ versus $\eta$, for the case of symmetrical efficiency $\eta=\eta_{a}=\eta_{b}$. The criterion for EPR steering is satisfied for $N=1$ provided $\eta>0.92$ but as expected for the NOON state, the cut-off efficiency increases sharply for higher $N$. For $N=2$ there is asymmetrical dependence on $\eta_{a}$ and $\eta_{b}$ as evident by the contour plots of Figure 2. The signature appears more sensitive to the efficiency $\eta_{B}$ of mode $b$. Such asymmetrical sensitivity depending on the steering direction has been noted previously 37, 38.

We note that the model 35 describes losses that occur prior to detection. It is assumed that the subsequent detection process gives no further loss. Alternatively, if the beam splitter is to model detection losses, then the losses would need to be assumed identical for each of the detection processes (number or homodyne). In reality, for low $N$ the numbers $n_{a}, n_{b}$ are usually detected via counting techniques where the efficiency of detection is often small. On the other hand, the quadratures $X$ and $P$ are measured via homodyne detection where efficiencies are high (at least for optical fields). This creates a

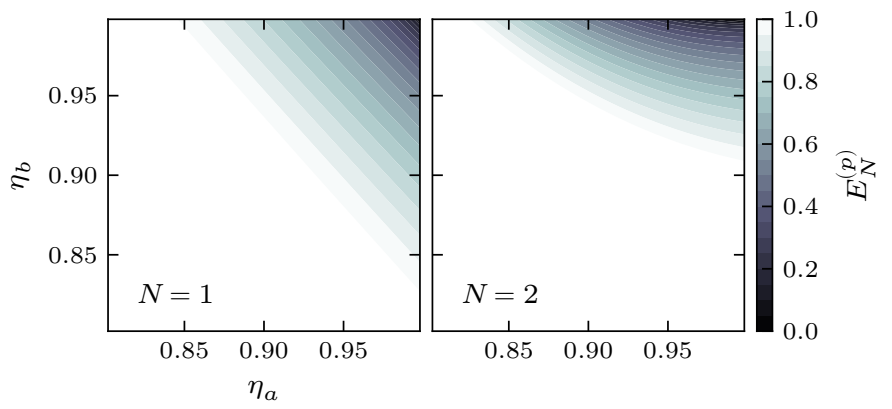

Figure 2: Contour plot shows the effect of loss on the EPR steering: EPR steering is observed when $E_{N}^{(p)}<1$. The $\eta_{a}$ and $\eta_{b}$ are the efficiencies for detection of mode $a$ and $b$ respectively.

situation where the loss coefficient $\eta$ is dependent on the choice of measurement made at each site, which we point out can create loopholes in the use of the signature for a practical experiment if not considered carefully [36, 39]. We discuss this further in the Conclusion.

\section{MEASUREMENT}

We next consider how to experimentally measure the moments on the right side of the steering inequalities (14) and 15 . For $N=1$ this is straightforward as explained in Section IV. For $N=2$, on examining the expressions (17) and 18, we see we need to measure $\left\langle\left[n, P^{2}\right]\right\rangle=$ $\langle X P+P X\rangle$. We define the measurable rotated quadrature phase amplitudes as $X_{\theta}=X \cos (\theta)+P \sin (\theta)$ and $P_{\theta}=-X \sin (\theta)+P \cos (\theta)$. Hence, $X_{\pi / 4}=\frac{1}{\sqrt{2}}\{X+P\}$ and $P_{\pi / 4}=\frac{1}{\sqrt{2}}\{-X+P\}$ and we note that $\left\langle X_{\pi / 4}^{2}\right\rangle=$ $\left\langle X^{2}+P^{2}+X P+P X\right\rangle / 2$. Thus, we can deduce either $\langle X P\rangle$ or $\langle P X\rangle$ by measuring the moments $\left\langle X^{2}\right\rangle,\left\langle P^{2}\right\rangle$ and $\left\langle X_{\pi / 4}^{2}\right\rangle$. The steering criteria 45 for $N=2$ can be written as (here we drop the subscripts $b$ for convenience)

$$
\begin{aligned}
\Delta_{i n f} n \Delta_{i n f}\left(P^{2}\right) & <\left|\left\langle\left[n, P^{2}\right]\right\rangle\right|_{i n f} / 2 \\
& =\left|\left\langle X_{\pi / 4}^{2}-X^{2} / 2-P^{2} / 2\right\rangle\right|_{i n f}
\end{aligned}
$$

and

$$
\begin{aligned}
\Delta_{i n f} n \Delta_{i n f}\left(X^{2}\right) & <\left|\left\langle\left[n, X^{2}\right]\right\rangle\right|_{i n f} / 2 \\
& =\left|\left\langle X_{\pi / 4}^{2}-X^{2} / 2-P^{2} / 2\right\rangle\right|_{i n f}
\end{aligned}
$$

The moments of $X, P$ and $X_{\pi / 4}$ are each measurable using homodyne detection.

For $N=3$, we see from $(17)$ and $(18)$ that we need to measure $\left[n, P^{3}\right]=3\left\langle P^{2} X+2 i P\right\rangle$ the other measurements 
being straightforward. Expanding gives

$$
\left\langle X_{\pi / 4}^{3}-P_{\pi / 4}^{3}\right\rangle=\frac{1}{\sqrt{2}}\left(\left\langle X^{3}\right\rangle+6 i\langle P\rangle+3\left\langle P^{2} X\right\rangle\right)
$$

Hence we can measure $\left\langle X_{\pi / 4}^{3}\right\rangle,\left\langle P_{\pi / 4}^{3}\right\rangle,\left\langle X^{3}\right\rangle,\langle P\rangle$ and consequently infer the value of $\left\langle P^{2} X\right\rangle$. Specifically, the steering inequalities become

$$
\begin{aligned}
\Delta_{i n f} n \Delta_{i n f}\left(P^{3}\right) & <\left|\left\langle\left[n, P^{3}\right]\right\rangle\right|_{i n f} / 2 \\
& =\left|\left\langle\sqrt{2}\left(X_{\pi / 4}^{3}-P_{\pi / 4}^{3}\right)-X^{3}\right\rangle\right|_{i n f} / 2
\end{aligned}
$$

and

$$
\begin{aligned}
\Delta_{i n f} n \Delta_{i n f}\left(X^{3}\right) & <\left|\left\langle\left[n, X^{3}\right]\right\rangle\right|_{i n f} / 2 \\
& =\left|\left\langle\sqrt{2}\left(X_{\pi / 4}^{3}+P_{\pi / 4}^{3}\right)-P^{3}\right\rangle\right|_{i n f} / 2
\end{aligned}
$$

We comment that the inequalities (37,40) are valid as a sufficiency test of EPR steering for all states i.e. we do not assume ideal NOON states.

\section{DISCUSSION}

To conclude, we discuss an obvious question, which is how to ensure in an experiment that the observed steering is due to the quantum coherence of the NOON superposition, as opposed to an alternative microscopic effect that might arise from superpositions of number states distinct by less than $N$ quanta? This is an important question where losses are present, because then the outcomes for number measurements can be different to 0 and $N$.

First, the answer is clear in the ideal case of a two-mode system that generates only outcomes 0 or $N$ for the number measurements. The density operator can then be written in terms of four basis states $|0\rangle|0\rangle,|0\rangle|N\rangle,|N\rangle|0\rangle,|N\rangle|N\rangle$. The violation of an EPR steering inequality is also confirmation of an entangled state, and in this case that can only imply entanglement involving the mesoscopically distinguishable basis states. The violation of the steering inequality confirms the presence of an $N$ th order off-diagonal matrix element (i.e. $\langle 0|\langle N|\rho| 0\rangle| N\rangle \neq 0)$. The details are straightforward and given in the Appendix E.

In experiments where loss or noise is present, the distribution $p_{n}$ for number $\hat{n}_{b}$ will include outcomes other than 0 and $N$. It is not then clear whether an observation of EPR steering is a result of the superposition of states such as $\left|M^{\prime}\right\rangle|n\rangle$ and $|M\rangle|m\rangle$ where $M-M^{\prime} \sim N$, or the result of less interesting superpositions where $M \sim M^{\prime}$.

The problem of determining whether the system has an $N$ th order quantum coherence (defined as $\langle 0|\langle N|\rho| 0\rangle| N\rangle \neq 0)$ is nontrivial [40 43]. However the following approach based on the steering inequality may be useful. The outcomes for number at mode $a$ are either $n_{a}>0$ or $n_{a}=0$. The distribution for the outcome $n_{b}$ of number at mode $b$ given any result $n_{a}>0$ is (for small losses) a "hill" centred near (or at) 0 . The distribution for $n_{b}$ given the result $n_{a}=0$ is a "hill centred near $N$. The mean and variance of each of the two hills is measurable and denoted by $\left\langle n_{b}\right\rangle_{1},\left(\Delta \hat{n}_{b}\right)_{1}^{2}$ and $\left\langle n_{b}\right\rangle_{2},\left(\Delta \hat{n}_{b}\right)_{2}^{2}$. For small losses, each of the two variances will be small.

We suppose that the experimentalist has measured a violation of the EPR steering inequality $\Delta_{i n f} \hat{n}_{b} \Delta_{i n f}\left(\hat{P}_{b}^{N}\right) \geq\left|\left\langle\hat{C}_{b}\right\rangle\right|_{\text {inf }} / 2$ where $\left(\Delta_{\text {inf }} \hat{n}_{b}\right)^{2}=$ $\sum_{n_{a}} P\left(n_{a}\right)\left(\Delta\left(n_{b} \mid n_{a}\right)\right)^{2}$ and $P\left(n_{a}\right)$ is the probability of outcome $n_{a}$. We note that where the conditional distribution for $n_{b}$ given $n_{a}$ is uniform for all $n_{a}>0$, this will imply violation of the new but similar inequality

$$
\left(P_{1}\left(\Delta \hat{n}_{b}\right)_{1}^{2}+P_{2}\left(\Delta \hat{n}_{b}\right)_{2}^{2}\right)\left(\Delta_{i n f} \hat{P}_{b}^{N}\right)^{2} \geq \frac{1}{4}\left|\left\langle\hat{C}_{b}\right\rangle\right|_{i n f}^{2}
$$

Here we specify as selected in (3) that the inferred values for $\hat{P}_{b}{ }^{N}$ and $\hat{C}_{b}$ are calculated using the same observable at mode $a$. Here $P_{1}$ is the probability of $n_{a}>0$ and we have assumed $\left(\Delta \hat{n}_{b}\right)_{1}^{2}=\Delta\left(n_{b} \mid n_{a}\right)$ for $n_{a}>0$. Similarly, $P_{2}$ is the probability that $n_{a}=0$ and $\left(\Delta \hat{n}_{b}\right)_{2}^{2}=\Delta\left(n_{b} \mid n_{a}\right)$ for $n_{a}=0$. We note that the loss model of Section VI predicts the distributions to be uniform, but if this is not the case then the inequality can be measured directly.

It is shown in the Appendix $F$ that violation of the inequality (41) is a negation of the mixture

$$
\rho=P_{1} \rho_{1}^{a b}+P_{2} \rho_{2}^{a b}
$$

where $\rho_{1}^{a b}$ and $\rho_{2}^{a b}$ are two-mode density operators with a mean and variance for $\hat{n}_{b}$ given by $\left\langle n_{b}\right\rangle_{1},\left(\Delta n_{b}\right)_{1}^{2}$ and $\left\langle n_{b}\right\rangle_{2},\left(\Delta n_{b}\right)_{2}^{2}$ respectively. The negation is for all mixtures of the form 42, which includes where $\rho_{i}^{a b}$ can be a superposition of number states. However the spread of number states involved in the superposition is constrained by the small variances associated with each $\rho_{i}^{a b}$. The $\rho_{1}^{a b}$ and $\rho_{2}^{a b}$ each have a variance for $\hat{n}_{b}$ that is narrower than the variance of the distribution given by the NOON superposition state. In other words, the violation of the inequality (41) can only be consistent with a density operator $\rho$ involving superpositions $\left|\psi_{\text {sup }}\right\rangle$ of states distributed over both hills.

\section{CONCLUSION}

The particular steering inequalities we present in this paper involve measurements of number as well as quadrature phase amplitude correlation. Number measurements often entail poor efficiencies. It would seem feasible to perform in the first instance an experiment based on post-selection of the events where a total of $N$ quanta (e.g. photons) are detected across both sites. The problem of distinguishing multiple from single photon counts 
at a given location require photon number-resolving detectors, or could be handled with $N$-photon counts being evaluated using multiple beam splitters [10, 13].

The experiment for $N=2$ would be a demonstration of a higher order (more mesoscopic) nonlocality than for $N=1$ and would seem not unrealistic given the high efficiencies available with homodyne detection. Our calculations show that $\eta>0.94$ is required. Care is needed to model the homodyne inefficiency as a loss before detection, and this small amount of loss must therefore also enter into the evaluation of the number correlation, to avoid the well-documented possible loopholes associated with losses that depend on measurement choices. The experiment for $N=1$ is feasible. Such an experiment would complement that performed recently by Fuwa et al [33] based on a different EPR steering inequality.

Finally, we point out that the steering inequalities (4 4 ) might be useful for detecting steering in other two-mode systems, especially where there is an inter-mode photon number correlation so that $\Delta_{i n f} n_{b}=0$. For instance, we can apply the first order inequality $\Delta_{i n f} \hat{n}_{b} \Delta_{i n f} \hat{P}_{b}<$ $\left|\left\langle\hat{X}_{b}\right\rangle\right|_{\text {inf }} / 2$ (Eq. (6) ) to the two-mode squeezed state. Denoting the two-mode squeeze parameter by $r$, the solutions for this state give $\Delta_{i n f} n_{b}=0$ for all $r$. Further, it is well known that there is an EPR correlation between the quadrature phase amplitudes of the two modes for all $r$ [25, 34, so that $\left|\left\langle\hat{X}_{b}\right\rangle\right|_{\text {inf }} \neq 0$ and $\Delta_{\text {inf }} \hat{P}_{b} \rightarrow 0$ as $r \rightarrow \infty$. While steering has been experimentally achieved for this state via the alternative EPR steering inequality $\Delta_{i n f} \hat{X}_{b} \Delta_{i n f} \hat{P}_{b}<1$ [34, 44, 45, it is quite possible that the use of the steering inequality with the number correlation $\Delta_{i n f} n_{b}=0$ (which is valid for all $r$ ) may provide advantages in some regimes.

\section{Acknowledgments}

We thank the Australian Research Council for support through the Discovery Project Grants scheme. We also thank Bryan Dalton, Peter Drummond and M. Chekhova for stimulating discussions about NOON states.

\section{Appendix A: Proof of Result (1)}

We will assume that the LHS model holds, for which moments are given by

$$
\begin{aligned}
\left\langle X_{A}(\theta) X_{B}(\phi)\right\rangle & =\int_{\lambda} d \lambda P(\lambda)\left\langle X_{A}(\theta)\right\rangle_{\lambda, \rho}\left\langle X_{B}(\phi)\right\rangle_{\lambda} \\
& \equiv \sum_{R} P_{R}\left\langle X_{A}(\theta)\right\rangle_{R, \rho}\left\langle X_{B}(\phi)\right\rangle_{R}
\end{aligned}
$$

Here we give two alternative (but equivalent) notations for the hidden variable-type parameters, denoting the continuous variable option by the symbol $\lambda$ as in Bell's work and the discrete option by $R$. The proof is unchanged whether we use integrals $(\lambda)$ or discrete summations $(R)$.

We consider the inference variance $\left(\Delta_{i n f} \sigma_{A}^{X}\right)^{2}$. Based on the definitions given in Section III, we see that $\sum_{x_{j}^{B}} P\left(x_{j}^{B}\right)\left\{\Delta\left(\sigma_{A}^{X} \mid x_{j}^{B}\right)\right\}^{2}=$ $\sum_{x_{j}^{B}} P\left(x_{j}^{B}\right) \sum_{\sigma_{A}^{X}} P\left(\sigma_{A}^{X} \mid x_{j}^{B}\right)\left\{\sigma_{A}^{X}-\left\langle\sigma_{A}^{X}\right\rangle_{x_{j}^{B}}\right\}^{2}$ which we can re-express as $\sum_{x_{j}^{B}, \sigma_{A}^{X}} P\left(x_{j}^{B}, \sigma_{A}^{X}\right)\left\{\sigma_{A}^{X}-\left\langle\sigma_{A}^{X}\right\rangle_{x_{j}^{B}}\right\}^{2}$ and hence as $\sum_{R} P_{R} \sum_{x_{j}^{B}, \sigma_{A}^{X}} P_{R}\left(x_{j}^{B}, \sigma_{A}^{X}\right)\left\{\sigma_{A}^{X}-\left\langle\sigma_{A}^{X}\right\rangle_{x_{j}^{B}}\right\}^{2}$. This follows using that for a probabilistic (hidden variable) mixture $P\left(x_{j}^{B}, \sigma_{X}^{A}\right)=\sum_{R} P_{R} P_{R}\left(x_{j}^{B}, \sigma_{X}^{A}\right)$. Now we note that $\left\langle(x-\delta)^{2}\right\rangle \geq\left\langle(x-\langle x\rangle)^{2}\right\rangle$ where $\delta$ is any number. Hence the expression becomes bounded from below, and we can simplify further to show that

$$
\begin{aligned}
& \sum_{R} P_{R} \sum_{x_{j}^{B}, \sigma_{A}^{X}} P_{R}\left(x_{j}^{B}, \sigma_{A}^{X}\right)\left\{\sigma_{A}^{X}-\left\langle\sigma_{A}^{X}\right\rangle_{x_{j}^{B}}\right\}^{2} \\
& \geq \sum_{R} P_{R} \sum_{x_{j}^{B}} P_{R}\left(x_{j}^{B}\right)\left\{\Delta_{R}\left(\sigma_{A}^{X} \mid x_{j}^{B}\right)\right\}^{2} \\
& =\sum_{R} P_{R}\left\{\Delta_{i n f, R} \sigma_{A}^{X}\right\}^{2}
\end{aligned}
$$

Here, the subscripts $R$ imply that the probabilities, averages and variances are with respect to the state $R$ and we have used that $\left\{\Delta_{R}\left(\sigma_{A}^{X} \mid x_{j}^{B}\right)\right\}^{2}=\sum_{\sigma_{A}^{X}} P_{R}\left(\sigma_{A}^{X} \mid x_{j}^{B}\right)\left\{\sigma_{A}^{X}-\right.$ $\left.\left\langle\sigma_{A}^{X}\right\rangle_{x_{j}^{B}, R}\right\}^{2}$. We note that the symbol $\lambda$ is used alternatively to $R$ in the main text, to describe that the variables may also be continuous. The proof follows similarly in either case. Now, if we assume the separability between the bipartition $A-B$ for each state $R$, in accordance with the LHS model (8), then

$$
P_{R}\left(x_{j}^{B}, \sigma_{A}^{X}\right)=P_{R}\left(x_{j}^{B}\right) P_{R}\left(\sigma_{A}^{X}\right)
$$

This implies $\left\langle\sigma_{A}^{X}\right\rangle_{x_{j}^{B}, R}=\left\langle\sigma_{A}^{X}\right\rangle_{R}$ and $\left\{\Delta_{R}\left(\sigma_{A}^{X} \mid x_{j}^{B}\right)\right\}^{2}=$ $\left(\Delta_{R} \sigma_{A}^{X}\right)^{2}$. Then we find, on using $\sum_{x_{j}^{B}} P_{R}\left(x_{j}^{B}\right)=1$, that we can write $\left\{\Delta_{i n f, R} \sigma_{A}^{X}\right\}^{2}=\left\{\Delta_{R} \sigma_{A}^{X}\right\}^{2}$. Thus, on applying the Cauchy-Schwarz inequality, we see that

$$
\begin{aligned}
\Delta_{\text {inf }}^{2} \sigma_{A}^{X} \Delta_{\text {inf }}^{2} \sigma_{A}^{Y} & \geq\left(\sum_{R} P_{R}\left\{\Delta_{R} \sigma_{A}^{X}\right\}^{2}\right)\left(\sum_{R} P_{R}\left\{\Delta_{R} \sigma_{A}^{Y}\right\}^{2}\right) \\
& \geq\left(\sum_{R} P_{R}\left\{\Delta_{R} \sigma_{A}^{X}\right\}\left\{\Delta_{R} \sigma_{A}^{Y}\right\}\right)^{2}
\end{aligned}
$$

where we define $\Delta_{i n f}^{2} \sigma_{A}^{X} \equiv\left(\Delta_{i n f} \sigma_{A}^{X}\right)^{2}$ and

$$
\Delta_{i n f}^{2} \sigma_{A}^{Y} \equiv\left(\Delta_{i n f} \sigma_{A}^{Y}\right)^{2}=\sum_{y_{j}^{B}} P\left(y_{j}^{B}\right)\left\{\Delta\left(\sigma_{A}^{X} \mid y_{j}^{B}\right)\right\}^{2}
$$

noting that the $\left\{y_{j}\right\}$ is the set of results for a measurement $y$ made at $B$ to infer the value of the measurement of $\sigma_{A}^{Y}$ at $A$. We consider an LHS model (8) where we assume the states at $A$ are local quantum states, so that we can use quantum uncertainty relations to derive 
a final steering inequality: e.g. $\left\{\Delta_{R}\left(\sigma_{A}^{X}\right)\right\}\left\{\Delta_{R}\left(\sigma_{A}^{Y}\right)\right\} \geq$ $\left|\left\langle\sigma_{A}^{Z}\right\rangle_{R}\right| / 2$ for any quantum state denoted by $R$. Using the above results, the LHS model implies

$$
\begin{aligned}
\left(\Delta_{i n f} \sigma_{A}^{X}\right)\left(\Delta_{i n f} \sigma_{A}^{Y}\right) & \geq \sum_{R} P_{R}\left\{\Delta_{R} \sigma_{A}^{X}\right\}\left\{\Delta_{R} \sigma_{A}^{Y}\right\} \\
& \geq \sum_{R} P_{R}\left(\left|\left\langle\sigma_{A}^{Z}\right\rangle_{R}\right| / 2\right)
\end{aligned}
$$

However, for a separable model, we know that $\left\langle\sigma_{A}^{Z}\right\rangle_{z_{j}^{B}, R}=\left\langle\sigma_{A}^{Z}\right\rangle_{R}$ and hence

$$
\begin{aligned}
\sum_{z_{j}^{B}} P\left(z_{j}^{B}\right) \sum_{R} P_{R}\left|\left\langle\sigma_{A}^{Z}\right\rangle_{z_{j}^{B}, R}\right| & =\sum_{R} P_{R} \sum_{z_{j}^{B}} P\left(z_{j}^{B}\right)\left|\left\langle\sigma_{A}^{Z}\right\rangle_{R}\right| \\
& =\sum_{R} P_{R}\left|\left\langle\sigma_{A}^{Z}\right\rangle_{R}\right|
\end{aligned}
$$

where here the $\left\{z_{j}\right\}$ is the set of results for a measurement $z$ at $B$, that we use to infer results for $\sigma_{A}^{Z}$. Hence

$$
\begin{aligned}
\left(\Delta_{i n f} \sigma_{A}^{X}\right)\left(\Delta_{i n f} \sigma_{A}^{Y}\right) & \geq \sum_{z_{j}^{B}} P\left(z_{j}^{B}\right) \sum_{R} P_{R}\left|\left\langle\sigma_{A}^{Z}\right\rangle_{z_{j}^{B}, R}\right| / 2 \\
& =\sum_{z_{j}^{B}} P\left(z_{j}^{B}\right)\left|\left\langle\sigma_{A}^{Z}\right\rangle_{z_{j}^{B}}\right| / 2
\end{aligned}
$$

We have used (for states constrained by the LHS model),

$$
\begin{aligned}
\left\langle\sigma_{A}^{Z}\right\rangle_{z_{j}^{B}} & =\sum_{\sigma_{A}^{Z}} \sigma_{A}^{Z} P\left(\sigma_{A}^{Z} \mid z_{j}^{B}\right) \\
& =\sum_{\sigma_{A}^{Z}} \sigma_{A}^{Z} \sum_{R} P_{R} P_{R}\left(\sigma_{A}^{Z} \mid z_{j}^{B}\right) \\
& =\sum_{R} P_{R}\left\langle\sigma_{A}^{Z}\right\rangle_{z_{j}^{B}, R}
\end{aligned}
$$

Defining $\left|\left\langle\sigma_{A}^{Z}\right\rangle\right|_{\text {inf }}=\sum_{z_{j}^{B}} P\left(z_{j}^{B}\right)\left|\left\langle\sigma_{A}^{Z}\right\rangle_{z_{j}^{B}}\right|$, we see finally that the LHS model implies $\left(\Delta_{\text {inf }} \sigma_{A}^{X}\right)\left(\Delta_{\text {inf }} \sigma_{A}^{Y}\right) \geq$ $\left|\left\langle\sigma_{A}^{Z}\right\rangle\right|_{\text {inf }} / 2$. Violation of this inequality implies failure of the LHS model, and therefore implies steering of $A$ by $B$. The result is steering of $B$ by $A$ if the $A$ and $B$ indices are exchanged (as in the main text). This completes the proof. $\square$

\section{Appendix B: Evaluation of inferred variances}

Here we will evaluate the inferred uncertainties $\Delta_{i n f}\left(X^{N}\right)$ and $\Delta_{i n f}\left(P^{N}\right)$ for the NOON state given in Eq. (1). We first consider $X \equiv X_{b}$ and evaluate $\Delta_{i n f}^{2}\left(X_{b}^{N}\right) \equiv\left(\Delta_{i n f}\left(X_{b}^{N}\right)\right)^{2}$, which is given by 22 . The terms of the form $\left\langle X_{b}^{n}\right\rangle_{i n f, x} \equiv\left\langle X_{b}^{n} \mid x\right\rangle$, with $n=N$ or $n=2 N$, are evaluated using the reduced density operator $\rho_{\text {red,x }}$ :

$$
\begin{aligned}
\rho_{\text {red }, x}= & \frac{1}{2}\left\{|\langle x \mid N\rangle|^{2}|0\rangle\left\langle 0\left|+e^{-i \phi}\langle x \mid N\rangle\langle 0 \mid x\rangle\right| 0\right\rangle\langle N|\right. \\
& +|\langle x \mid 0\rangle|^{2}|N\rangle\left\langle N\left|+e^{i \phi}\langle x \mid 0\rangle\langle N \mid x\rangle\right| N\right\rangle\langle 0|(\zeta \mathrm{B} 1)
\end{aligned}
$$

and the fact that operators $\hat{X}$ and $\hat{P}$ can be described in terms of a complete set of projectors as $\hat{X}_{B}^{n}=$ $\int_{-\infty}^{\infty} x_{B}^{n}\left|x_{B}\right\rangle\left\langle x_{B}\right| d x_{B}$ and $\hat{P}_{B}^{n}=\int_{-\infty}^{\infty} p_{B}^{n}\left|p_{B}\right\rangle\left\langle p_{B}\right| d p_{B}$. Therefore we get:

$$
\begin{aligned}
\left\langle X^{n}\right\rangle_{\text {inf }, x}= & \operatorname{Tr}\left(\rho_{\text {red }, x} X^{n}\right) \\
= & \frac{1}{2 P(x)}\left[|\langle x \mid N\rangle|^{2} \int x_{B}^{n}\left|\left\langle x_{B} \mid 0\right\rangle\right|^{2} d x_{B}\right. \\
& +e^{-i \phi}\langle x \mid N\rangle\langle 0 \mid x\rangle \int x_{B}^{n}\left\langle N \mid x_{B}\right\rangle\left\langle x_{B} \mid 0\right\rangle d x_{B} \\
& +e^{i \phi}\langle x \mid 0\rangle\langle N \mid x\rangle \int x_{B}^{n}\left\langle 0 \mid x_{B}\right\rangle\left\langle x_{B} \mid N\right\rangle d x_{B} \\
& \left.+|\langle x \mid 0\rangle|^{2} \int x_{B}^{n}\left|\left\langle x_{B} \mid N\right\rangle\right|^{2} d x_{B}\right]
\end{aligned}
$$

where $P(x)=\frac{1}{2}\left[|\langle x \mid 0\rangle|^{2}+|\langle x \mid N\rangle|^{2}\right]$ is the probability of measuring $X_{A}$ and getting outcome $x$ and $\langle x \mid N\rangle$ are the harmonic oscillator functions given in Eq. (27). The value for $\Delta_{i n f}\left(X_{b}^{N}\right)$ is obtained on evaluating the expressions of $\left\langle X^{n}\right\rangle_{\text {inf, } x}$, with $n=N$ or $2 N$, and substituting on the expression given in Eq. 222. Similarly we evaluate the inferred variance of $P \equiv P_{b}$, which is given by 21). Using the reduced density operator $\rho_{\text {red,x }}$ given above we find:

$$
\begin{aligned}
\left\langle P^{n}\right\rangle_{\text {inf, } x}= & \frac{1}{2 P(x)}\left[|\langle x \mid N\rangle|^{2} \int p_{B}^{n}\left|\left\langle p_{B} \mid 0\right\rangle\right|^{2} d p_{B}\right. \\
& +e^{-i \phi}\langle x \mid N\rangle\langle 0 \mid x\rangle \int p_{B}^{n}\left\langle 0 \mid p_{B}\right\rangle\left\langle p_{B} \mid N\right\rangle d p_{B} \\
& +e^{i \phi}\langle x \mid 0\rangle\langle N \mid x\rangle \int p_{B}^{n}\left\langle N \mid p_{B}\right\rangle\left\langle p_{B} \mid 0\right\rangle d p_{B} \\
& \left.+|\langle x \mid 0\rangle|^{2} \int p_{B}^{n}\left|\left\langle p_{B} \mid N\right\rangle\right|^{2} d p_{B}\right]
\end{aligned}
$$

To evaluate, we first consider $N=2$. We let $\phi=\pi / 2$ :

$$
\begin{aligned}
\left\langle X^{2}\right\rangle_{\text {inf }, x}= & \frac{1}{2 P(x)}\left[|\langle x \mid 2\rangle|^{2} \int x_{B}^{2}\left|\left\langle x_{B} \mid 0\right\rangle\right|^{2} d x_{B}\right. \\
& \left.+|\langle x \mid 0\rangle|^{2} \int x_{B}^{2}\left|\left\langle x_{B} \mid 2\right\rangle\right|^{2} d x_{B}\right] \\
= & 1+\frac{8}{3-2 x^{2}+x^{4}}
\end{aligned}
$$

and

$$
\left\langle X^{4}\right\rangle_{\text {inf }, x}=3+\frac{72}{x^{4}-2 x^{2}+3}
$$

where we have used that $P(x)=\frac{e^{-\frac{x^{2}}{2}}}{2 \sqrt{2 \pi}}\left(\frac{\left(2 x^{2}-2\right)^{2}}{8}+1\right)$. On performing the integration using the above results we get for the $N=2$ state that $\Delta_{i n f}^{2}\left(X_{b}^{2}\right)=10.1351$ and $\Delta_{i n f}\left(X_{b}^{2}\right)=3.18356$. Similarly we evaluate $\Delta_{i n f}^{2}\left(P_{b}^{N}\right)$ :

$$
\begin{aligned}
\left\langle P^{2}\right\rangle_{\text {inf }, x} & =1+\frac{8}{3-2 x^{2}+x^{4}} \\
\left\langle P^{4}\right\rangle_{\text {inf }, x} & =3+\frac{72}{x^{4}-2 x^{2}+3}
\end{aligned}
$$


These results are the same as for $X$, since for this value of angle $e^{i \phi}=i=-e^{-i \phi}$, and also

$$
\begin{aligned}
\int p_{B}^{n}\left\langle 0 \mid p_{B}\right\rangle\left\langle p_{B} \mid 2\right\rangle d p_{B} & =\int p_{B}^{n}\left\langle 2 \mid p_{B}\right\rangle\left\langle p_{B} \mid 0\right\rangle d p_{B} \\
& =\int x_{B}^{n}\left\langle 0 \mid x_{B}\right\rangle\left\langle x_{B} \mid 2\right\rangle d x_{B}
\end{aligned}
$$

so that the second and third terms of equations (B3) cancel. We obtain for $N=2$ that $\Delta_{\text {inf }}^{2}\left(P_{b}^{2}\right)=10.1351$.

Continuing for higher $N$, we obtain for $N=3$ $\Delta_{\text {inf }}^{2}\left(P_{b}^{3}\right)=477.081$ and $\Delta_{\text {inf }}\left(P_{b}^{3}\right)=21.8422$; for $N=4$, $\Delta_{\text {inf }}^{2}\left(P_{b}^{4}\right)=10982.8$ and $\Delta_{\text {inf }}\left(P_{b}^{4}\right)=104.799$; and for $N=5, \Delta_{\text {inf }}^{2}\left(P_{b}^{5}\right)=795639$ and $\Delta_{\text {inf }}\left(P_{b}^{5}\right)=891.986$. Identical results are obtained for the inferred variances in $X^{N}$.

\section{Appendix C: Evaluation of $\left|\left\langle\left[n_{b}, X_{b}^{N}\right]\right\rangle\right|_{\text {inf }}$ and $\left|\left\langle\left[n_{b}, P_{b}^{N}\right]\right\rangle\right|_{i n f}$}

The expressions for the terms $\left|\left\langle\left[n_{b}, X_{b}^{N}\right]\right\rangle\right|_{\text {inf }}$ and $\left|\left\langle\left[n_{b}, P_{b}^{N}\right]\right\rangle\right|_{\text {inf }}$ are calculated from Eq. 16 and the first line of Eq. (18). Using that $X_{b}=b+b^{\dagger}$ and $P_{b}=\left(b-b^{\dagger}\right) / i$, we note that on evaluating the expectation value for the NOON states given in Eq. (1), the only nonzero contributions involve terms of the form $\left\langle b^{N}\right\rangle$ and $\left\langle b^{\dagger N}\right\rangle$ :

$$
\begin{aligned}
& \left|\left\langle\left[n_{b}, X_{b}^{N}\right]\right\rangle\right|_{i n f}=N\left|-\left\langle b^{N}\right\rangle+\left\langle b^{\dagger N}\right\rangle\right|_{i n f} \\
& \left|\left\langle\left[n_{b}, P_{b}^{N}\right]\right\rangle\right|_{i n f}=N\left|\left\langle b^{N}\right\rangle+(-1)^{N+1}\left\langle b^{\dagger N}\right\rangle\right|_{i n f}
\end{aligned}
$$

We evaluate $\left\langle b^{N}\right\rangle=\operatorname{Tr}\left[\rho_{\text {red }, x} b^{N}\right]$ and $\left\langle b^{\dagger N}\right\rangle=$ $\operatorname{Tr}\left[\rho_{\text {red,x }}\left(b^{\dagger}\right)^{N}\right]$ using the reduced density matrix given in Eq. B1):

$$
\begin{aligned}
\left\langle\hat{b}^{N}\right\rangle_{\text {inf,x }} & =\frac{e^{i \phi}}{2 P(x)} \sqrt{N !}\langle x \mid 0\rangle\langle x \mid N\rangle \\
\left\langle\left(\hat{b}^{\dagger}\right)^{N}\right\rangle_{\text {inf }, x} & =\frac{e^{-i \phi}}{2 P(x)} \sqrt{N !}\langle x \mid 0\rangle\langle x \mid N\rangle
\end{aligned}
$$

On integrating over all possible values we get:

$$
\left|\left\langle\left[n_{b}, X_{b}^{N}\right]\right\rangle\right|_{i n f}=N \sqrt{N !}|\sin \phi| \int_{-\infty}^{\infty}|\langle x \mid N\rangle\langle 0 \mid x\rangle| d x
$$

For $\left|\left\langle\left[n_{b}, P_{b}^{N}\right]\right\rangle\right|_{\text {inf }}$ the expression for $N$ odd is given by:

$$
\left|\left\langle\left[n_{b}, P_{b}^{N}\right]\right\rangle\right|_{i n f}=N \sqrt{N !}|\cos \phi| \int_{-\infty}^{\infty}|\langle x \mid N\rangle\langle 0 \mid x\rangle| d x
$$

while for $N$ even we find:

$$
\left|\left\langle\left[n_{b}, P_{b}^{N}\right]\right\rangle\right|_{i n f}=N \sqrt{N !}|\sin \phi| \int_{-\infty}^{\infty}|\langle x \mid N\rangle\langle 0 \mid x\rangle| d x
$$

We obtain for $N=2$ that $\left|\left\langle\left[n_{b}, P_{b}^{N}\right]\right\rangle\right|_{\text {inf }}=1.93577$ with $\phi=\pi / 2$; for $N=3,\left|\left\langle\left[n_{b}, P_{b}^{N}\right]\right\rangle\right|_{\text {inf }}=4.53$ with $\phi=0$; for $N=4,\left|\left\langle\left[n_{b}, P_{b}^{N}\right]\right\rangle\right|_{i n f}=11.2024$ with $\phi=\pi / 2$; and for $N=5,\left|\left\langle\left[n_{b}, P_{b}^{N}\right]\right\rangle\right|_{i n f}=29.5504$ with $\phi=0$.

\section{Appendix D: Including losses}

The detected fields $\hat{a}_{\text {det }}, \hat{b}_{\text {det }}$ are given by

$$
\begin{aligned}
a_{\text {det }} & =\sqrt{\eta_{a}} a+\sqrt{1-\eta_{a}} a_{v} \\
a_{\text {loss }} & =-\sqrt{1-\eta_{a}} a+\sqrt{\eta_{a}} a_{v}
\end{aligned}
$$

with similar definitions for the mode operators $b_{\text {det }}$ and $b_{\text {loss }}$. Using these transformations it is possible to write the operators $a, b$ and hence the NOON state $|\psi\rangle$ of Eq. (1) in terms of $a_{d e t}^{\dagger}, a_{\text {loss }}^{\dagger}, b_{d e t}^{\dagger}$ and $b_{l o s s}^{\dagger}$. We will denote the vacuum state for all four modes by $|0\rangle$. The density operator $\rho=|\psi\rangle\langle\psi|$ can then also be expressed in terms of these operators. Since we are not interested in the modes $a_{\text {loss }}$ and $b_{\text {loss }}$ (which we label $A$,loss and $B$,loss) we take the trace over the states of the loss mode to evaluate $\rho^{\prime} \equiv \operatorname{Tr}_{A, l o s s ; B, \operatorname{loss}} \rho$. After using the binomial expansion for terms such as $\left(\sqrt{\eta_{a}} a_{d e t}^{\dagger}-\sqrt{\left(1-\eta_{a}\right)} a_{l o s s}^{\dagger}\right)$ and performing the trace, the reduced density operator for the detected modes is:

$$
\begin{aligned}
\rho^{\prime} & =\frac{1}{2}\left[\sum_{s}\left(\begin{array}{c}
N \\
N-s
\end{array}\right)\left(\eta_{a}\right)^{N-s}\left(1-\eta_{a}\right)^{s}|N-s\rangle_{A, \operatorname{det}}\langle N-s|\otimes| 0\rangle_{B, \operatorname{det}}\left\langle 0\left|+\left(\sqrt{\eta_{a} \eta_{b}}\right)^{N} e^{-i \phi}\right| N\right\rangle_{A, \text { det }}\langle 0|\otimes| 0\rangle_{B, \operatorname{det}}\langle N|\right. \\
& \left.+\left(\sqrt{\eta_{a} \eta_{b}}\right)^{N} e^{i \phi}|0\rangle_{A, \operatorname{det}}\langle N|\otimes| N\rangle_{B, \operatorname{det}}\left\langle 0\left|+\sum_{s}\left(\begin{array}{c}
N \\
N-s
\end{array}\right)\left(\eta_{b}\right)^{N-s}\left(1-\eta_{b}\right)^{s}\right| 0\right\rangle_{A, \operatorname{det}}\langle 0|\otimes| N-s\rangle_{B, \text { det }}\langle N-s|\right](\mathrm{D} 1)
\end{aligned}
$$


1. Calculating $\Delta_{\text {inf }}^{2}\left(P_{b}^{N}\right)$ and $\Delta_{i n f}^{2}\left(X_{b}^{N}\right)$

The $\Delta_{i n f}^{2}\left(P_{b}^{N}\right)$ and $\Delta_{i n f}^{2}\left(X_{b}^{N}\right)$ are the inferred variances of quantities $P_{b}^{N}$ and $X_{b}^{N}$ due to a measurement in $X_{a}$. These are given by (21) and (22). We evaluate these inferred variances using the density operator for modes $a_{d e t}$ and $b_{d e t}$ given in Eq. (D1). For the inferred variances we evaluate the density operator $\rho^{\prime \prime}$, where we consider that the mode $A$, det is in the state $|x\rangle$. This density operator is given by:

$$
\begin{aligned}
\rho^{\prime \prime}= & \frac{|x\rangle_{A, d e t}\left\langle x\left|\rho^{\prime}\right| x\right\rangle_{A, d e t}\langle x|}{P(x)} \\
= & \frac{1}{2 P(x)}\left[\sum_{s}\left(\begin{array}{c}
N \\
N-s
\end{array}\right)\left(\eta_{a}\right)^{N-s}\left(1-\eta_{a}\right)^{s}\langle x \mid N-s\rangle_{A d}\langle N-s \mid x\rangle|x\rangle_{A d}\langle x|\otimes| 0\rangle_{B d}\langle 0|\right. \\
& +\left(\sqrt{\eta_{a} \eta_{b}}\right)^{N} e^{-i \phi}\langle x \mid N\rangle_{A d}\langle 0 \mid x\rangle|x\rangle_{A d}\langle x|\otimes| 0\rangle_{B d}\left\langle N\left|+\left(\sqrt{\eta_{a} \eta_{b}}\right)^{N} e^{i \phi}\langle x \mid 0\rangle_{A d}\langle N \mid x\rangle\right| x\right\rangle_{A d}\langle x|\otimes| N\rangle_{B d}\langle 0| \\
& \left.+\sum_{s}\left(\begin{array}{c}
N \\
N-s
\end{array}\right)\left(\eta_{b}\right)^{N-s}\left(1-\eta_{b}\right)^{s}\langle x \mid 0\rangle_{A d}\langle 0 \mid x\rangle|x\rangle_{A d}\langle x|\otimes| N-s\rangle_{B d}\langle N-s|\right]
\end{aligned}
$$

where

$$
\begin{aligned}
P(x)= & \operatorname{Tr}\left[|x\rangle_{A, \text { det }}\left\langle x\left|\rho^{\prime}\right| x\right\rangle_{A, \text { det }}\langle x|\right] \\
= & \frac{1}{2}\left[\sum_{s}\left(\begin{array}{c}
N \\
N-s
\end{array}\right)\left(\eta_{a}\right)^{N-s}\left(1-\eta_{a}\right)^{s}|\langle x \mid N-s\rangle|^{2}\right. \\
& \left.+\sum_{s}\left(\begin{array}{c}
N \\
N-s
\end{array}\right)\left(\eta_{b}\right)^{N-s}\left(1-\eta_{b}\right)^{s}|\langle x \mid 0\rangle|^{2}\right] \quad(\mathrm{D} 2)
\end{aligned}
$$

Here we are using the following notation for the modes: $A d \equiv A$, det and $B d \equiv B$, det. In order to compute $\Delta^{2}\left(P_{b}^{N} \mid x\right)$ and $\Delta^{2}\left(X_{b}^{N} \mid x\right)$, we trace out the $A$, det mode to get the reduced density operator for $B$, det mode:

$$
\begin{aligned}
\rho_{\text {red,det }, x}= & \operatorname{Tr}_{A, \text { det }}\left(\rho^{\prime \prime}\right) \\
= & \frac{1}{2 P(x)}\left[\sum_{s}\left(\begin{array}{c}
N \\
N-s
\end{array}\right)\left(\eta_{a}\right)^{N-s}\left(1-\eta_{a}\right)^{s}\langle x \mid N-s\rangle_{A d}\langle N-s \mid x\rangle|0\rangle_{B d}\langle 0|\right. \\
& +\left(\sqrt{\eta_{a} \eta_{b}}\right)^{N} e^{-i \phi}\langle x \mid N\rangle_{A d}\langle 0 \mid x\rangle|0\rangle_{B d}\left\langle N\left|+\left(\sqrt{\eta_{a} \eta_{b}}\right)^{N} e^{i \phi}\langle x \mid 0\rangle_{A d}\langle N \mid x\rangle\right| N\right\rangle_{B d}\langle 0| \\
& \left.+\sum_{s}\left(\begin{array}{c}
N \\
N-s
\end{array}\right)\left(\eta_{b}\right)^{N-s}\left(1-\eta_{b}\right)^{s}\langle x \mid 0\rangle_{A d}\langle 0 \mid x\rangle|N-s\rangle_{B d}\langle N-s|\right]
\end{aligned}
$$

The inferred variances are defined as:

$$
\begin{aligned}
\Delta^{2}\left(X_{b}^{N} \mid x\right) & =\left\langle\left(X_{b}^{N}\right)^{2} \mid x\right\rangle-\left\langle X_{b}^{N} \mid x\right\rangle^{2} \\
\Delta^{2}\left(P_{b}^{N} \mid x\right) & =\left\langle\left(P_{b}^{N}\right)^{2} \mid x\right\rangle-\left\langle P_{b}^{N} \mid x\right\rangle^{2}
\end{aligned}
$$




$$
\begin{aligned}
\left\langle X_{b}^{n} \mid x\right\rangle & =\frac{1}{2 P(x)}\left[\sum_{s}\left(\begin{array}{c}
N \\
N-s
\end{array}\right)\left(\eta_{a}\right)^{N-s}\left(1-\eta_{a}\right)^{s}\langle x \mid N-s\rangle_{A d}\langle N-s \mid x\rangle \int x_{B}^{n}\left\langle 0 \mid x_{B}\right\rangle\left\langle x_{B} \mid 0\right\rangle d x_{B}\right. \\
& +\left(\sqrt{\eta_{a} \eta_{b}}\right)^{N} e^{-i \phi}\langle x \mid N\rangle_{A d}\langle 0 \mid x\rangle \int x_{B}^{n}\left\langle N \mid x_{B}\right\rangle\left\langle x_{B} \mid 0\right\rangle d x_{B}+\left(\sqrt{\eta_{a} \eta_{b}}\right)^{N} e^{i \phi}\langle x \mid 0\rangle_{A d}\langle N \mid x\rangle \int x_{B}^{n}\left\langle 0 \mid x_{B}\right\rangle\left\langle x_{B} \mid N\right\rangle d x_{B} \\
& \left.+\sum_{s}\left(\begin{array}{c}
N \\
N-s
\end{array}\right)\left(\eta_{b}\right)^{N-s}\left(1-\eta_{b}\right)^{s}\langle x \mid 0\rangle_{A d}\langle 0 \mid x\rangle \int x_{B}^{n}\left\langle N-s \mid x_{B}\right\rangle\left\langle x_{B} \mid N-s\right\rangle d x_{B}\right]
\end{aligned}
$$

$$
\begin{aligned}
\left\langle P^{n}\right\rangle_{\text {inf }, x} & =\frac{1}{2 P(x)}\left[\sum_{s}\left(\begin{array}{c}
N \\
N-s
\end{array}\right)\left(\eta_{a}\right)^{N-s}\left(1-\eta_{a}\right)^{s}\langle x \mid N-s\rangle_{A, d e t}\langle N-s \mid x\rangle \int p_{B}^{n}\left\langle 0 \mid p_{B}\right\rangle\left\langle p_{B} \mid 0\right\rangle d p_{B}\right. \\
& +\left(\sqrt{\eta_{a} \eta_{b}}\right)^{N} e^{-i \phi}\langle x \mid N\rangle_{A d}\langle 0 \mid x\rangle \int p_{B}^{n}\left\langle N \mid p_{B}\right\rangle\left\langle p_{B} \mid 0\right\rangle d p_{B}+\left(\sqrt{\eta_{a} \eta_{b}}\right)^{N} e^{i \phi}\langle x \mid 0\rangle_{A d}\langle N \mid x\rangle \int p_{B}^{n}\left\langle 0 \mid p_{B}\right\rangle\left\langle p_{B} \mid N\right\rangle d p_{B} \\
& \left.+\sum_{s}\left(\begin{array}{c}
N \\
N-s
\end{array}\right)\left(\eta_{b}\right)^{N-s}\left(1-\eta_{b}\right)^{s}\langle x \mid 0\rangle_{A, d e t}\langle 0 \mid x\rangle \int p_{B}^{n}\left\langle N-s \mid p_{B}\right\rangle\left\langle p_{B} \mid N-s\right\rangle d p_{B}\right]
\end{aligned}
$$

The value of the corresponding variances for $\Delta^{2}\left(X_{b}^{N} \mid x\right)$ and $\Delta^{2}\left(P_{b}^{N} \mid x\right)$ of equations $\left.\mathrm{D} 4\right)$ is evaluated using the expressions given in equations (D5) and (D6) considering $n=N$ or $n=2 N$.

\section{Inferred variances $\Delta_{i n f}^{2}\left(n_{b}\right)$ including losses}

$\Delta_{\text {inf }}^{2}\left(n_{b}\right)$ is the inferred variance of $n_{b}$ due to a measurement in $n_{a}$. In order to evaluate this variance we will consider that the outcome in $n_{a}$ is $m$. We define $P(m)$ as the probability for obtaining the result $m$ for $n_{a}$. Next, we evaluate the reduced density operator $\rho_{m}$ for the modes $A$, det and $B$, det given that the outcome is $m$ :

$$
\begin{aligned}
\rho_{m}= & \frac{1}{P(m)}\left[|m\rangle_{A d}\left\langle m\left|\rho^{\prime}\right| m\right\rangle_{A d}\langle m|\right] \\
= & {\left[\left(\begin{array}{c}
N \\
m
\end{array}\right) \eta_{a}^{m}\left(1-\eta_{a}\right)^{N-m}|m\rangle_{A d}\langle m|\otimes| 0\rangle_{B d}\langle 0|\right.} \\
& +\sum_{s}\left(\begin{array}{c}
N \\
N-s
\end{array}\right) \eta_{b}^{N-s}\left(1-\eta_{b}\right)^{s} \\
& \left.\times|0\rangle_{A d}\langle 0|\otimes| N-s\rangle_{B d}\langle N-s|\right] /(2 P(m))
\end{aligned}
$$

where

$$
\begin{aligned}
P(m) & =\operatorname{Tr}\left[|m\rangle_{A, \operatorname{det}}\left\langle m\left|\rho^{\prime}\right| m\right\rangle_{A, \operatorname{det}}\langle m|\right] \\
& =\frac{1}{2}\left(\begin{array}{c}
N \\
m
\end{array}\right) \eta_{a}^{m}\left(1-\eta_{a}\right)^{N-m}+\frac{1}{2}
\end{aligned}
$$

In order to write the last line we have used that $\sum_{s}^{N}\left(\begin{array}{c}N \\ N-s\end{array}\right) \eta_{b}^{N-s}\left(1-\eta_{b}\right)^{s}=1$.

Next we evaluate $\left\langle n_{B}\right\rangle_{\text {inf }, m}=\operatorname{Tr}\left[\rho_{m} n_{B}\right]$ and $\left\langle n_{B}^{2}\right\rangle_{\text {inf }, m}=\operatorname{Tr}\left[\rho_{m} n_{B}^{2}\right]$ obtaining:

$$
\begin{aligned}
\left\langle n_{B}\right\rangle_{\text {inf }, m} & =\frac{1}{2} \frac{\sum_{s}\left(\begin{array}{c}
N \\
N-s
\end{array}\right) \eta_{b}\left(1-\eta_{b}\right)^{s} \delta_{m, 0}(N-s)}{P\left(n_{A}=m\right)} \\
\left\langle n_{B}^{2}\right\rangle_{\text {inf }, m} & =\frac{1}{2} \frac{\sum_{s}\left(\begin{array}{c}
N \\
N-s
\end{array}\right) \eta_{b}\left(1-\eta_{b}\right)^{s} \delta_{m, 0}(N-s)^{2}}{P\left(n_{A}=m\right)}
\end{aligned}
$$

Since $n_{A}=m=0$ is the only non-zero contribution for the statistical moments we obtain:

$$
\begin{aligned}
\left\langle n_{B}\right\rangle_{\text {inf }, 0} & =\frac{1}{2} \frac{N \eta_{b}}{P\left(n_{A}=0\right)} \\
\left\langle n_{B}^{2}\right\rangle_{\text {inf }, 0} & =\frac{1}{2} \frac{\eta_{b}\left(N-N \eta_{b}+N^{2} \eta_{b}\right)}{P\left(n_{A}=0\right)} \\
P\left(n_{A}=0\right) & =\frac{1}{2}\left(\left(1-\eta_{a}\right)^{N}+1\right)
\end{aligned}
$$

Using the above results we evaluate the inferred variance for $m=0$, which we denote by $\Delta_{i n f}^{2} n_{b, 0}$ :

$$
\Delta_{i n f}^{2} n_{b, 0}=\frac{\eta_{b}\left(N-N \eta_{b}\right)+N \eta_{b}\left(1-\eta_{a}\right)^{N}\left(1-\eta_{b}+N \eta_{b}\right)}{\left(\left(1-\eta_{a}\right)^{N}+1\right)^{2}}
$$


In order to evaluate the variance of the inferred value $n_{B}$, we sum over all possible values of $m$ obtaining:

$$
\begin{aligned}
\Delta^{2} n_{\text {inf }} & =\sum_{m}^{N} P\left(n_{A}=0\right) \Delta^{2} n_{\text {inf }, m=0} \\
& =\frac{\eta_{b}\left(N-N \eta_{b}\right)+N\left(1-\eta_{a}\right)^{N}\left(\eta_{b}-\eta_{b}^{2}+N \eta_{b}^{2}\right)}{2\left(\left(1-\eta_{a}\right)^{N}+1\right)}
\end{aligned}
$$

\section{Evaluation of $\left|\left\langle\left[n_{b}, X_{b}^{N}\right]\right\rangle\right|_{i n f}$ and $\left|\left\langle\left[n_{b}, P_{b}^{N}\right]\right\rangle\right|_{\text {inf }}$}

Full evaluation of the terms $\left|\left\langle\left[n_{b}, X_{b}^{N}\right]\right\rangle\right|_{\text {inf }}$ and $\left|\left\langle\left[n_{b}, P_{b}^{N}\right]\right\rangle\right|_{\text {inf }}$ (given by Eqs. (16) and 18p) reveals that for the lossy system and for $N \leq 5$ :

$$
\begin{aligned}
& \left|\left\langle\left[n_{b}, X_{b}^{N}\right]\right\rangle\right|_{\text {inf }}=N\left|-\left\langle b^{N}\right\rangle+\left\langle b^{\dagger N}\right\rangle\right|_{\text {inf }} \\
& \left|\left\langle\left[n_{b}, P_{b}^{N}\right]\right\rangle\right|_{\text {inf }}=N\left|\left\langle b^{N}\right\rangle+(-1)^{N+1}\left\langle b^{\dagger N}\right\rangle\right|_{\text {inf }}
\end{aligned}
$$

We evaluate $\left\langle b^{N}\right\rangle=\operatorname{Tr}\left[\rho_{\text {red,det }, x} b^{N}\right]$ and $\left\langle b^{\dagger N}\right\rangle=$ $\operatorname{Tr}\left[\rho_{\text {red,det, } x}\left(b^{\dagger}\right)^{N}\right]$ using the reduced density matrix given in Eq. (D3) and performed the corresponding trace, we obtain

$$
\left\langle b^{N}\right\rangle_{\text {inf }, x}=\frac{1}{2 P(x)}\left(\sqrt{\eta_{a} \eta_{b}}\right)^{N} e^{i \phi}\langle x \mid N\rangle\langle 0 \mid x\rangle \sqrt{N !}
$$

and $\left\langle\left(b^{\dagger}\right)^{N}\right\rangle_{\text {inf, } x}=\left(\left\langle b^{N}\right\rangle_{\text {inf }, x}\right)^{*}$. Thus the expressions obtained are identical to $\mathrm{C} 2 \mathrm{C} 3$ but replacing $\sqrt{N \text { ! with }}$ $C_{\eta}=\sqrt{N !}\left(\sqrt{\eta_{a} \eta_{b}}\right)^{N}$.

\section{Appendix E: Proof of $N$ th order quantum coherence and entanglement}

The question is how to prove experimentally that the system is indeed in a superposition of the two number states $|N\rangle|0\rangle$ and $|0\rangle|N\rangle$ that are distinct by $N$ quanta in each mode. For such a state, the density matrix $\rho$ has a nonzero off-diagonal element:

$$
\langle 0|\langle N|\rho| 0\rangle| N\rangle \neq 0
$$

We refer to the nonzero term (E1) as an $N$ th order quantum coherence. The presence of this term distinguishes the superposition of the two states $|N\rangle|0\rangle,|0\rangle|N\rangle$ from a classical mixture of the two states.

In the ideal scenario, the experiment generates only outcomes 0 or $N$ for the number measurements $n_{a}$ or $n_{b}$. It is then straightforward to show that any violation of an EPR steering inequality is also a signature of an $N$ th order quantum coherence (E1). The objective is to construct the density operator $\rho$ for the system and to prove that necessarily (E1) holds. Since there are only two outcomes for each mode, any viable two-mode density operator could be written in terms of four basis states $|0\rangle|0\rangle,|0\rangle|N\rangle,|N\rangle|0\rangle,|N\rangle|N\rangle$. Supposing an EPR steering inequality to be violated, this will negate the LHS model given by (8) and therefore also any fully separable quantum model

$$
\rho=\sum_{R} P_{R} \rho_{a} \rho_{b}
$$

where $\rho_{a}$ and $\rho_{b}$ are density matrices for the single modes $a$ and $b$ 22. Thus the system cannot be in any mixture of the basis states $|0\rangle|0\rangle,|0\rangle|N\rangle,|N\rangle|0\rangle,|N\rangle|N\rangle$ which are separable states. There are only certain remaining possibilities for $\rho$ and these require non-zero off-diagonal elements. For example, for the NOON state the results for number measurements $\hat{n}$ would be either 0 or $N$ in one mode, correlated with $N$ or 0 in the other mode. Assuming that there is a nonzero probability for the outcome for $|0\rangle|N\rangle$ and $|N\rangle|0\rangle$, this ensures that the off-diagonal term $\langle 0|\langle N|\rho| 0\rangle| N\rangle$ is nonzero. Also, the failure of the separable model (E2) ensures the system cannot be in the product state $(|N\rangle+|0\rangle)(|N\rangle+|0\rangle) / 2$. This implies the system is the entangled superposition of states $|0\rangle|N\rangle$ and $|N\rangle|0\rangle$.

\section{Appendix F: Proof of inequality 41}

First we prove the the uncertainty relation

$$
\left(\Delta \hat{n}_{b}\right)^{2}\left(\Delta_{i n f} \hat{P}_{b}^{N}\right)^{2} \geq \frac{1}{4}\left|\left\langle\hat{C}_{b}\right\rangle\right|_{i n f}^{2}
$$

which holds for any two-mode state. We follow the methods used in Refs. 41. The variance is defined as $\left(\Delta \hat{n}_{b}\right)^{2}=\sum_{n_{b}} P\left(n_{b}\right)\left(n_{b}-\left\langle n_{b}\right\rangle\right)^{2}$ (denoting the outcomes of $\hat{n}_{b}$ by $\left.n_{b}\right)$. We can consider marginals and joint distributions for the measurements on both modes $a$ and $b$. Thus we write $\left(\Delta \hat{n}_{b}\right)^{2}=\sum_{n_{b}, p_{a}} P\left(n_{b}, p_{a}\right)\left(n_{b}-\left\langle n_{b}\right\rangle\right)^{2}$ and then $\left(\Delta \hat{n}_{b}\right)^{2}=\sum_{n_{b}, p_{a}} P\left(n_{b} \mid p_{a}\right) P\left(p_{a}\right)\left(n_{b}-\left\langle n_{b}\right\rangle\right)^{2}$. Thus

$$
\begin{aligned}
\left(\Delta \hat{n}_{b}\right)^{2} & =\sum_{p_{a}} P\left(p_{a}\right) \sum_{n_{b}} P\left(n_{b} \mid p_{a}\right)\left(n_{b}-\left\langle n_{b}\right\rangle\right)^{2} \\
& \geq \sum_{p_{a}} P\left(p_{a}\right) \sum_{n_{a}} P\left(n_{b} \mid p_{a}\right)\left(n_{b}-\left\langle n_{b}\right\rangle_{p_{a}}\right)^{2} \\
& =\sum_{p_{a}} P\left(p_{a}\right)\left(\Delta\left(n_{b} \mid p_{a}\right)\right)^{2}
\end{aligned}
$$

where $\left(\Delta\left(n_{b} \mid p_{a}\right)\right)^{2}=\sum_{n_{a}} P\left(n_{b} \mid p_{a}\right)\left(n_{b}-\left\langle n_{b}\right\rangle_{p_{a}}\right)^{2}$ and $\left\langle n_{b}\right\rangle_{p_{a}}$ is the mean of the conditional distribution $P\left(n_{b} \mid p_{a}\right)$. For each $p_{a}$ we have defined the distribution $P\left(n_{b} \mid p_{a}\right)$ as $P_{p_{a}}\left(n_{b}\right)$ and we see that the quantity $\sum_{n_{b}} P_{p_{a}}\left(n_{b}\right)\left(n_{b}-X\right)^{2}$ where $X$ is any constant, is minimised by the choice $X=\left\langle n_{b}\right\rangle_{p_{a}}=\sum_{n_{b}} P_{p_{a}}\left(n_{b}\right) n_{b}$. Next, we write 


$$
\begin{aligned}
\left(\Delta \hat{n}_{b}\right)^{2}\left(\Delta_{i n f} \hat{P}_{b}^{N}\right)^{2} \geq & \left\{\sum_{p_{a}} P\left(p_{a}\right)\left(\Delta\left(n_{b} \mid p_{a}\right)\right)^{2}\right\} \\
& \left\{\sum_{p_{a}} P\left(p_{a}\right)\left(\Delta\left(P_{b}^{N} \mid p_{a}\right)^{2}\right\}\right. \\
\geq & \left|\sum_{p_{a}} P\left(p_{a}\right) \Delta\left(n_{b} \mid p_{a}\right) \Delta\left(P_{b}^{N} \mid p_{a}\right)\right|^{2} \\
\geq & \frac{1}{4}\left|\sum_{p_{a}} P\left(p_{a}\right)\right|\left\langle C_{b}\right\rangle_{p_{a}} \|^{2}=\frac{1}{4}\left|\left\langle\hat{C}_{b}\right\rangle\right|_{\text {inf }}^{2}
\end{aligned}
$$

where we apply the Cauchy-Schwarz inequality and use the uncertainty relation (2) that holds for the state of $b$ conditioned on the measurement result $p_{a}$ of mode $a$. This proves (F1). Then we can say that for the mixture $\rho^{a b}$ of 42 (using that for a mixture it is true that [46] $(\Delta O)_{\rho}^{2} \geq \sum_{i} P_{i}(\Delta O)_{i}^{2}$ where $O$ is any quantum observable, and also true that $\left(\Delta_{\text {inf }} P_{b}^{N}\right)^{2} \geq \sum_{i} P_{i}\left(\Delta_{i n f} P_{b}^{N}\right)_{i}^{2}$ [4]):

$$
\begin{aligned}
\left\{\sum_{i} P_{i}\left(\Delta \hat{n}_{b}\right)_{i}^{2}\right\}\left(\Delta_{i n f} \hat{P}_{b}^{N}\right)^{2} & \geq\left\{\sum_{i} P_{i}\left(\Delta n_{b}\right)_{i}^{2}\right\} \\
& \left\{\sum_{i} P_{i}\left(\Delta_{i n f} P_{b}^{N}\right)_{i}^{2}\right\} \\
& \geq\left|\sum_{i} P_{i}\left(\Delta n_{b}\right)_{i}\left(\Delta_{i n f} P_{b}^{N}\right)_{i}\right|^{2} \\
& \geq\left.\left.\frac{1}{4}\left|\sum_{i} P_{i}\right|\left\langle\hat{C}_{b}\right\rangle\right|_{i n f, i}\right|^{2} \quad(\mathrm{~F} 2)
\end{aligned}
$$

where we use the Cauchy-Schwarz inequality and that the uncertainty relation (2) holds for each $\rho_{i}^{a b}$. Now we see that $\sum_{p_{a}} P\left(p_{a}\right)\left|\left\langle C_{b}\right\rangle_{p_{a}}\right|=\sum_{p_{a}} P\left(p_{a}\right)\left|\sum_{c_{b}} C_{b} P\left(C_{b} \mid p_{a}\right)\right|$. If the system is described by the mixture $\rho^{a b}$ then

$$
\begin{aligned}
\left\langle C_{b}\right\rangle_{p_{a}} & =\sum_{c_{b}} C_{b} P\left(C_{b} \mid p_{a}\right) \\
& =\sum_{C_{b}} C_{b} \frac{P\left(C_{b}, p_{a}\right)}{P\left(p_{a}\right)} \\
& =\sum_{C_{b}} C_{b} \sum_{i} P_{i} \frac{P_{i}\left(C_{b}, p_{a}\right)}{P\left(p_{a}\right)} \\
& =\sum_{i} P_{i} \sum_{C_{b}} C_{b} \frac{P_{i}\left(p_{a}\right)}{P\left(p_{a}\right)} P_{i}\left(C_{b} \mid p_{a}\right)
\end{aligned}
$$

$$
\begin{aligned}
\left|\sum_{i} P_{i} \sum_{C_{b}} C_{b} \frac{P_{i}\left(p_{a}\right)}{P\left(p_{a}\right)} P_{i}\left(C_{b} \mid p_{a}\right)\right| \leq & \sum_{i} P_{i} \frac{P_{i}\left(p_{a}\right)}{P\left(p_{a}\right)} \\
& \times\left|\sum_{C_{b}} C_{b} P_{i}\left(C_{b} \mid p_{a}\right)\right|
\end{aligned}
$$

Thus from F3

where the subscript $i$ denotes the probabilities for the component $\rho_{i}^{a b}$. We can write

$$
\begin{aligned}
\sum_{p_{a}} P\left(p_{a}\right)\left|\left\langle C_{b}\right\rangle_{p_{a}}\right| & \leq \sum_{i} P_{i} \sum_{p_{a}} P_{i}\left(p_{a}\right)\left|\sum_{C_{b}} C_{b} P_{i}\left(C_{b} \mid p_{a}\right)\right| \\
& =\sum_{i} P_{i}\left|\left\langle\hat{C}_{b}\right\rangle\right|_{i n f, i}
\end{aligned}
$$

where $\left|\left\langle\hat{C}_{b}\right\rangle\right|_{i n f, i}=\sum_{p_{a}} P_{i}\left(p_{a}\right)\left|\sum_{C_{b}} C_{b} P_{i}\left(C_{b} \mid p_{a}\right)\right|$. Thus we have proved that $\sum_{i} P_{i}\left|\left\langle\hat{C}_{b}\right\rangle\right|_{\text {inf }, i} \geq$ $\sum_{p_{a}} P\left(p_{a}\right)\left|\left\langle C_{b}\right\rangle_{p_{a}}\right|$. Hence we can write from $(\mathrm{F} 2)$ :

$$
\begin{aligned}
\left\{\sum_{i} P_{i}\left(\Delta n_{b}\right)_{i}^{2}\right\}\left(\Delta_{i n f} P_{b}^{N}\right)^{2} & \geq \frac{1}{4}\left|\sum_{i} P_{i}\right|\left\langle\hat{C}_{b}\right\rangle\left|{ }_{i n f, i}\right|^{2} \\
& \geq \frac{1}{4}\left|\sum_{p_{a}} P\left(p_{a}\right)\right|\left\langle C_{b}\right\rangle_{p_{a}} \|^{2} \\
& =\left.\frac{1}{4}\left|\left\langle\hat{C}_{b}\right\rangle\right|_{i n f}\right|^{2}
\end{aligned}
$$

This proves the inequality 41.
[1] E. Schroedinger, Naturwiss. 23, 807 (1935).

[2] W. H. Zurek, Physics Today 44 (10), 36-44 (1991). A. O. Caldeira and A. J. Leggett, Phys. Rev. A 31, 1059 (1985). D. F. Walls, and G. J. Milburn, Phys. Rev. A 31, 2403 (1985).

[3] B. Yurke and D. Stoler, Phys. Rev. Lett. 57, 13 (1986).

[4] M. Brune et al., Phys. Rev. Lett. 77, 4887 (1996). C. Monroe et al., Science 272, 1131 (1996). J. Friedman et al., Nature 40643 (2000). D. Leibfried et al., Nature
438, 639 (2005). A. Ourjoumtsev et al., Nature 448, 784 (2007).

[5] C. K. Hong, Z. Y. Ou, and L. Mandel, Phys. Rev. Lett. 59, 2044 (1987). Z. Y. Ou, L. J Wang, X. Y. Zou and L. Mandel, Phys. Rev. A 41, 566 (1990); ibid Phys. Rev. A 42, 2957 (1990).

[6] J. P. Dowling, Contemporary Physics 49, 125 (2008).

[7] Hwang Lee, Pieter Kok and Jonathan P. Dowling, Journal of Modern Optics 49, 2325 (2002). P. Kok, H. Lee, 
and J. P. Dowling, Phys. Rev. A 65, 052104 (2002). J. Fiurášek, Phys. Rev. A 65, 053818 (2002).

[8] P. Walther, J.-W. Pan, M. Aspelmeyer, R. Ursin, S. Gasparoni, and A. Zeilinger, Nature 429, 158-161 (2004).

[9] M. W. Mitchell, J. S. Lundeen and A. M. Steinberg, Nature 429, 161 (2004).

[10] I. Afek, O. Ambar and Y. Silberberg, Science 328, 879 (2010).

[11] Y. Israel, I. Afek, S. Rosen, O. Ambar and Y. Silberberg, Phys. Rev. A 85, 022115 (2012).

[12] H. Kim, H. S. Park, S.-K. Choi, Opt. Express 17, 19720 (2009).

[13] R. Kruse, L. Sansoni, S. Brauner, R. Ricken, C. S. Hamilton, I. Jex, and C. Silberhorn, Phys. Rev. A 92, 053841 (2015). J. Lugani, S. Ghosh, and K. Thyagarajan, Phys. Rev. A 83, 062333 (2011).

[14] A. Boto et al. Phys. Rev. Lett. 85, 2733 (2000). Y.-H. Kim and Y. H. Shih, Found. Phys. 29, 1849 (1999).

[15] M. Angelo, M. Chekhova and Y. Shih, Phys. Rev. Lett. 87, 13602 (2001).

[16] R. W. Boyd and J. P. Dowling, Quantum Information Processing 11, 891 (2012).

[17] R. J. Lewis-Swan and K. V. Kheruntsyan, Nat. Commun. 5, 3752 (2014). S. S. Hodgman, R. G. Dall, A. G. Manning, K. G. H. Baldwin, A. G. Truscott, Science, 331 (6020), 1046 (2011).

[18] G. J. Milburn, J. Corney, E. M. Wright and D. F. Walls, Phys. Rev. A 55, 4318 (1997). J. I. Cirac, M. Lewenstein, K. Molmer, and P. Zoller, Phys. Rev. A 57, 1208 (1998). D. M. Gordan and C. M. Savage, Phys. Rev. A 59, 4623, (1999). J. Dunningham and K. Burnett, Journ. Modern Optics, 48, 1837, (2001). C. Lee, Phys. Rev. Lett. 97, 150402 (2006). T. J. Haigh, A. J. Ferris, and M. K Olsen, Opt. Commun. 283, 3540 (2010). L. D. Carr, D. R. Dounas-Frazer, and M. A. Garcia-March, Europhys. Lett. 90, 10005 (2010). H. Cable, F. Laloë and W. J. Mullin, Phys. Rev. A 83, 053626 (2011). Q. Y. He, P. D. Drummond, M. K. Olsen and M. D. Reid, Phys. Rev. A 86, 023626 (2012).

[19] See Special Issue on "Macroscopic quantumness: theory and applications in optical sciences", Optics Communications, pp 1-110, 337, (2015).

[20] B. Hensen et al., Nature 526, 682 (2015); M. Giustina et al., 115, 250401 Phys. Rev. Lett. (2015); L.K. Shalm et al., Phys. Rev. Lett. 115, 250402 (2015).

[21] N. D. Mermin, Phys. Rev. D 22, 356 (1980). P. D. Drummond, Phys. Rev. Lett. 50, 1407 (1983). M. Zukowski, Phys. Lett. A 177, 290 (1993). A. Peres, Found. Phys. 29, 589 (1999). H. Weinfurter and M. Zukowski, Phys. Rev. A 64, 010102 (2001). M. D. Reid, Phys. Rev. Lett. 84, 2765 (2000). M. Zukowski, and C. Brukner, Phys. Rev. Lett. 88, 210401 (2002). D. Collins et al., Phys. Rev. Lett. 88, 040404 (2002). M. D. Reid, W. J. Munro, and F. De Martini, Phys. Rev. A 66, 033801 (2002). M. Eibl et al., Phys. Rev. Lett. 90, 200403 (2003). A. LamasLinares, J. Howell \& D. Bouwmeester, Nature 412, 887 (2001).

[22] H. M. Wiseman, S. J. Jones, and A. C. Doherty, Phys. Rev. Lett. 98, 140402 (2007). S. J. Jones, H. M. Wiseman, A. C Doherty, Phys. Rev. A 76, 052116 (2007).

[23] E. G. Cavalcanti, S. J. Jones, H. M. Wiseman, M. D.
Reid, Phys. Rev. A 80, 032112 (2009).

[24] D. Smith et al., Nat. Commun. 3, 625 (2012). B. Wittmann et al., New J. Phys. 14, 053030 (2012). A. J. Bennet et al., Phys. Rev. X 2, 031003 (2012).

[25] M. D. Reid et al., Rev. Mod. Phys. 81, 1727 (2009).

[26] A. Einstein, B. Podolsky, and N. Rosen, Phys. Rev. 47, 777 (1935).

[27] E. Schrödinger, Proc. Cambridge Philos. Soc. 31, 555 (1935); 32, 446 (1936).

[28] Max Born, Born-Einstein Letters, 1916-1955: Friendship, Politics and Physics in Uncertain Times (Palgrave MacMillan, 2005).

[29] P. Chowdhury, T. Pramanik, A. S. Majumdar, and G. S. Agarwal, Phys. Rev. A 89, 012104 (2014). P. Chowdhury, T. Pramanik and A. S. Majumdar, Phys. Rev. A 92, 042317 (2015)

[30] C. Wildfeuer, A. Lund and J. Dowling, Phys. Rev. A 76, 052101 (2007). F. Toppel, M. V. Chekhova and G. Leuchs, arXiv:1607.01296 (2016). I. Kogias, P. Skrzypczyk, D. Cavalcanti, A. Acin and G. Adesso, Phys. Rev. Lett. 115, 210401 (2015).

[31] S. M. Tan, D. F. Walls, and M. J. Collett, Phys. Rev. Lett. 66, 252 (1991). L. Hardy, Phys. Rev. Lett. 73, 2279 (1994). J. Dunningham and V. Vedral, Phys. Rev. Lett. 99, 180404 (2007). S. A. Babichev, J. Appel, and A. I. Lvovsky, Phys. Rev. Lett. 92, 193601 (2004).

[32] S. Jones and H.M. Wiseman, Phys. Rev. A 84, 012110 (2011).

[33] M. Fuwa, S. Takeda, M. Zwierz, H. M. Wiseman, and A. Furusawa, Nat. Commun. 6, 6665 (2015).

[34] M. D. Reid, Phys. Rev A 40, 913 (1989).

[35] M. D. Reid, Phys.Rev. A 88, 062338 (2013).

[36] J. S. Bell, Physics 1, 195 (1964). J. F. Clauser and A. Shimony, Rep. Prog. Phys. 41, 1881 (1978). N. Brunner et al., Rev. Mod. Phys. 86, 419 (2014).

[37] Q. Y. He and M. D. Reid, Phys. Rev. A 88, 052121 (2013). S. Kiesewetter et al., Phys. Rev. A 90043805 (2014). L. Rosales-Zárate et al., J. Opt. Soc. Am. B 32 A82 (2015).

[38] S. L. W. Midgley, A. J. Ferris, and M. K. Olsen, Phys. Rev. A 81, 022101 (2010). V. Händchen et al., Nature Photon. 6, 596 (2012).

[39] P. Pearle, Phys. Rev. D 2, 1418 (1970).

[40] E. G. Cavalcanti and M. D. Reid, Phys. Rev. Lett. 97, 170405 (2006). C. Marquardt et al., Phys. Rev. A 76 030101 (2007).

[41] E. G. Cavalcanti and M. D. Reid, Phys Rev A 77, 062108 (2008). B. Opanchuk, L. Rosales-Zárate, R. Y. Teh and M. D. Reid, arXiv:1609.06028 (2016)..

[42] B. Yadin and V. Vedral, Phys. Rev. A 93, 022122 (2016).

[43] F. Fröwis, P. Sekatski, P. Pavel and W. Dür, Phys. Rev. Lett. 116090801 (2016).

[44] Z. Y. Ou et al., Phys. Rev. Lett. 68, 3663 (1992).

[45] M. D. Reid and P. D Drummond, Phys. Rev. Lett. 60, 2731 (1988).

[46] H. Hofmann and S. Takeuchi, Phys. Rev.A 68, 032103 (2003).

[47] M. J. Mallon, M. D. Reid and M. K. Olsen, J. Phys. B: At. Mol. Opt. Phys, 41, 015501 (2008). 